\title{
Serine Palmitoyltransferase Controls Stemness of Intestinal Progenitors
}

Ying $\mathrm{Li}^{1 \dagger}$, Bhagirath Chaurasia ${ }^{1 * \dagger}$, Vincent Kaddai ${ }^{1}$, Joseph L. Wilkerson ${ }^{1}$, J. Alan Maschek ${ }^{1}$, James $\mathrm{Cox}^{2}$, Peng Wei ${ }^{2}$, Claire Bensard ${ }^{2}$, Peter J Meikle ${ }^{3}$, Hans Clevers ${ }^{4}$, James A Shayman ${ }^{5}$, Yoshio Hirabayashi ${ }^{6}$, William L. Holland ${ }^{1}$, Jared Rutter ${ }^{2,7}$, Scott A. Summers ${ }^{1 *}$

\section{Affiliations}

1. Department of Nutrition and Integrative Physiology and the Diabetes and Metabolism Research Center, University of Utah, 15 North 2030 East, Salt Lake City, UT 84112

2. Department of Biochemistry and the Diabetes and Metabolism Research Center, University of Utah, Salt Lake City, UT 84112

3. Baker Heart and Diabetes Institute, Melbourne, Australia

4. Hubrecht Institute, Royal Netherlands Academy of Arts and Sciences (KNAW) and UMC Utrecht, 3584 CT Utrecht, the Netherlands; Oncode Institute, Hubrecht Institute, 3584 CT Utrecht, the Netherlands; The Princess Maxima Center for Pediatric Oncology, 3584 CS Utrecht, the Netherlands

5. Department of Internal Medicine, University of Michigan, Ann Arbor, Michigan 48109, USA

6. Cellular Informatics Laboratory RIKEN Cluster for Pioneering Research, Wako-Shi, Saitama Japan

7. Howard Hughes Medical Institute, Salt Lake City, UT 84112

\section{$\dagger$ Authors contributed equally}

\author{
*Address for correspondence \\ Scott Summers, PhD \\ Department of Nutrition and Integrative Physiology and the Diabetes and Metabolism Research \\ Center, University of Utah, 15 North 2030 East, Salt Lake City, UT 84112 \\ Email: Scott.a.summers@,health.utah.edu \\ Bhagirath Chaurasia, $\mathrm{PhD}$ \\ Department of Nutrition and Integrative Physiology and the Diabetes and Metabolism Research \\ Center, University of Utah, 15 North 2030 East, Salt Lake City, UT 84112 \\ E-mail: bhagirath.chaurasia@health.utah.edu
}

Keywords: Stem cell, sphingolipids, ceramides, metabolism, fatty acid uptake, fatty acid binding protein, colorectal cancer 


\section{Summary}

Cancers of the gastrointestinal tract including esophageal adenocarcinomas, colorectal cancers, and cancers of the gastric cardia are common comorbidities of obesity. Excessive delivery of macronutrients to the cells lining the gut can increase one's risk for these cancer by inducing imbalances in the rate of intestinal stem cell proliferation vs. differentiation, which can produce polyps and other aberrant growths. We demonstrate that serine palmitoyltransferase (SPT), which diverts dietary fatty and amino acids into the sphingolipid biosynthesis pathway, is a critical modulator of intestinal stem cell homeostasis. SPT and other enzymes in the biosynthetic pathway are upregulated in human colon tumors. These enzymes produce sphingolipids that serve as prostemness signals that stimulate peroxisome-proliferator activated receptor alpha (PPAR $\alpha$ )mediated induction of fatty acid binding protein-1. This increases fatty acid uptake and oxidation and enhances the stemness program. Serine palmitoyltransferase thus serves as a critical link between dietary macronutrients, epithelial regeneration, and cancer risk.

\section{Introduction}

The intestinal epithelium is the most rapidly regenerating tissue in the body. Constant mechanical damage inflicted by passing bowel content necessitates the nearly complete renewal of the epithelium every 4-5 days (Leblond and Stevens, 1948). Replacement of the intestinal villi results from a coordinated process triggered by the proliferation and differentiation of the intestinal stem cells (ISCs) that lie in a crypt at the base of the villus. The ISCs give rise to the specialized absorptive cells (i.e. enterocytes) and mucus and hormone-secreting goblet and enteroendocrine cells, respectively, that comprise the bulk of the villus epithelium. The balance between ISC proliferation and differentiation is tightly regulated and its disruption leads to gastroenteritis or hyperproliferative lesions and tumors.

ISCs respond to nutritional cues that increase proliferation and enhance the regenerative capacity of the gut epithelium. For example, the fatty acid palmitate stimulates proliferation of ISCs, thus increasing the number of cells within the crypt to enhance tumor risk (Beyaz et al., 2016). By contrast, serine deprivation restricts growth of intestinal cancers (Maddocks et al., 2017). Since palmitate and serine are required to synthesize the sphingoid backbone of sphingolipids, we developed the hypothesis tested herein that one or more sphingolipids might be important signaling intermediates linking these dietary macronutrients to stem cell fate.

Once palmitate enters cells, it is quickly conjugated to coenzyme-A, which traps the fatty acid in the cell and activates it for subsequent metabolism. The resultant palmitoyl-CoA can be coupled to (i) a glycerol backbone to produce glycerophospholipids, (ii) carnitine for transport into mitochondria, or (iii) the aforementioned amino acid serine to generate sphingolipids. This latter serine conjugation step is catalyzed by serine palmitoyltransferase (SPT), which generates the sphingoid backbone present in sphingolipids. This moiety subsequently acquires additional, variable fatty acids and a critical double bond to form ceramides, which are the precursors of sphingomyelins and gangliosides (Figure 1a). Ceramides, particularly those containing a C16-acyl chain, have emerged as important nutrient signals that alter cellular metabolism in response to excessive ectopic fatty acids (Summers et al., 2019). Though sphingolipids are relatively minor components (Figure 1B, light blue) of the mouse gut lipidome that are far less abundant than glycerolipids and sterols (Figure 1B, dark blue), the studies presented herein reveal that they are 
potent nutrient signals that alter metabolism of ISCs to enhance stemness and catalyze epithelial regeneration.

\section{Results}

Sphingolipids and sphingolipid-synthesizing enzymes are upregulated in human colon cancer

Adenocarcinomas that were surgically removed from colon cancer patients (Table 1) contained higher levels of $\mathrm{C}_{16}$-ceramides, $\mathrm{C}_{16}$-dihydroceramides, and sphingosine than the healthy tissue surrounding the lesion (Figure 1C). They also contained higher levels of transcripts encoding two critical SPT subunits (SPTLC1\&2) and several other sphingolipid-synthesizing enzymes (i.e. CERS6, DEGS1\&2, and SGMS1\&2) (Figures 1D). CERS6 encodes the ceramide synthase that is required to make the $\mathrm{C}_{16}$-ceramides that are signals of nutritional excess (Hammerschmidt et al., 2019; Raichur et al., 2019; Raichur et al., 2014; Turpin et al., 2014) and are elevated in the human tumors (Figure 1C). By comparison, the other CERS isoforms (1-5), and the ceramides they produce, were unaltered in the lesions (Figure 1C and S1B). The SGMS transcripts that encode the sphingomyelin synthases that convert ceramide into sphingomyelin were slightly elevated, but sphingomyelins were not (Figure S1A). Moreover, other genes that influence ceramide synthesis or metabolism were unaltered (Figure S1B). Thus, the increase in transcripts and sphingolipids was largely restricted to the enzymes in the de novo synthesis pathway that produce the $\mathrm{C}_{16^{-}}$ ceramides. We also quantified two glycerol-containing lipids (i.e. diacylglycerol and triacylglycerol), finding that they were reduced in the tumors (Figure 1E). This finding suggests that these intestinal tumors redirect fatty acids away from the glycerolipid pathway and towards sphingolipids. As expected, the tumors contained substantially higher levels of $L G R 5$, a marker of ISCs (Figure S1C).

Excision of Sptlc2 from mouse intestines decreases ceramides and other sphingolipid biosynthetic intermediates within the intestinal crypts

To better understand the role of sphingolipids in the gut, we produced conditional knockout mice that allow for acute depletion of the Sptlc2 subunit of SPT from intestinal epithelial cells (IECs). These $S p t l c 2^{\delta I E C}$ mice were generated by breeding $S p t l c 2^{f l / f l}$ mice that we described previously (Chaurasia et al., 2016) with mice expressing a tamoxifen inducible Cre-recombinase controlled by the villin promoter (Tg(Vil-cre/ERT2)(Goh et al., 2015). Within two days of tamoxifen administration, adult $S p t l c 2^{\delta I E C}$ mice displayed a marked reduction in Sptlc2 mRNA expression in the small intestine and colon, as compared to tamoxifen-treated Sptlc $2^{f l f l}$ littermates (Figure 2A). The depletion of Sptlc2 reduced levels of the sphingolipids in the de novo synthesis pathway (i.e. ceramides, dihydroceramides, sphinganine, and phytoceramides) within the intestinal crypts (Figure S2A). Surprisingly, it did not alter levels of complex sphingolipids (i.e. sphingomyelins and glucosylceramides) (Figure S2A). The effects of Sptlc2 depletion manifest predominantly in the crypts, and not the villi; tamoxifen-treatment only affected dihydroceramides, phytoceramides and sphinganine, but not other sphingolipids, within villi of the $S p t l c 2^{\delta I E C}$ mice (Figure S2B).

Excision of Sptlc2 from mouse intestines disrupts gut architecture and depletes proliferating ISCs Deletion of Sptlc2 led to rapid weight loss (Figure 2B) and death of all animals within 4 days (Figure S2F). Upon necropsy, the $S p t l c 2^{\delta I E C}$ mice were found to have a short small intestine (Figure 2C and Figure S2C) and a small spleen (Figure S2D). The intestine exhibited increased 
permeability (Figure 2D), inflammation (Figure S2E). Treatment with broad-spectrum antibiotics prolonged animal survival (Figure S2F), suggesting that sepsis resulting from loss of the intestinal barrier was the ultimate cause of death of the animals. Careful histological assessment revealed severe disruption of the small intestine characterized by disorganized villi and nearly complete loss of crypts (Figure 2E). Following the four-day tamoxifen regimen, the $S p t l c 2^{\delta I E C}$ mice showed alterations in many cell-types within the villus structure; Goblet cells (PAS), Paneth cells (LYZ1) and ISCs (OLFM4) were depleted and/or mis-localized (Figure 2F). Immunohistochemistry with anti-Ki67 antibodies also revealed a stark decrease in the rapidly proliferating cells located within the crypt (Figure 2F).

To identify which cell types were first affected by Sptlc2 depletion, we conducted a temporal assessment of the changing pathology, collecting the jejunum through each of the first three days following tamoxifen administration. Though the gross epithelial structure of small intestine in the $S p t l c 2^{\delta I E C}$ mice remained relatively intact through the first three treatment days, the ISC pool started to disappear within 2-days of tamoxifen injection. This finding was evidenced by reduced expression of OLFM4 (Figure 2G), a disproportionate reduction in the number of crypts (Figure 2H), and fewer proliferating cells in the crypts as assessed by Ki67 staining (Figure S2G and $\mathrm{S} 2 \mathrm{H})$.

$\underline{\text { Excision of Sptlc2 from ISCs recapitulates the lethal phenotype of the Sptlc }{ }^{\delta I E C} \text { mice }}$

The findings presented thus far suggest that sphingolipids might be autonomous and essential signals that control the proliferation of ISCs in order to promote epithelial regeneration. Experiments using RNA in situ hybridization reinforced this idea, as they revealed that Sptlc2 transcript levels were much higher in the crypts than they were in the villi (Figure 2I). This observation strongly suggests that the ISCs within the crypt were the major site of sphingolipid synthesis and action. This conclusion was corroborated by the lipidomic assessments showing higher ceramide and phytoceramide levels in the crypts, as compared to the villi (Figure S3A). To selectively study the role of ISC sphingolipids, we generated a mouse line allowing for ISCspecific Sptlc2 depletion (Sptlc2 $2^{\delta I S C}$ ) by breeding the aforementioned Sptlc2 flfl mice with Olfm4EGFP-ires-CreERT2 mice (Schuijers et al., 2014). Injecting tamoxifen into the Sptlc2 ${ }^{\delta I S C}$ mice led to depletion of Sptlc2 from the crypts, while it was retained in the other cells in the villi (Figure 2I). This led to quick depletion of the ISCs, as assessed by labeling Olfm4 using RNA in situ hybridization (Figure 2I). By comparison, Lyzl, which demarcates Paneth cells in the crypt, was largely unaffected by Sptlc2 depletion (Figure 2I). In these Sptlc2 ${ }^{\delta I S C}$ mice, tamoxifen also recapitulated the gross phenotype of the $S p t l c 2^{\delta I E C}$ mice, including the reduction in body weight, rapid death, short small intestine and small spleen (Figure S3B-S3F). Histologic analysis showed a comparable disruption of the small intestine structure that was most pronounced in the crypts (Figure 2J).

Restoring sphingolipids enhances stemness and promotes intestinal organoid survival

To better understand how SPT regulates stem cells, we turned to an intestinal organoid 3D culture system (Sato et al., 2009). These mini-intestines contain all of the cell types of the mature epithelium but allow for precise control of the tissue environment. Pharmacological inhibition of SPT (i.e. treatment with the SPT inhibitor myriocin) or genetic ablation of Sptlc2 [i.e. treatment with 4-hydroxytamoxifen (4OHT)] led to rapid death of the organoids, as assessed by propidium iodide staining (Figure 3A). Thus, we recapitulated the in vivo phenotype caused by Sptlc2 depletion using this in vitro system. Remarkably, viability of the organoids could be restored by 
supplementing with short-chain analogs of ceramide $\left(\mathrm{C}_{2}\right.$-ceramide, $\left.25 \mu \mathrm{M}\right)$ (Figure $\left.3 \mathrm{~A}\right)$. Mass spectroscopy revealed that $\mathrm{C}_{2}$-ceramide restored levels of many sphingolipids in either the myriocin or $4 \mathrm{OHT}$ treated organoids (e.g. ceramides, glucosylceramides, sphingomyelins and sphingosine), excepting those sphingolipids that cannot be re-produced from $\mathrm{C}_{2}$-ceramide via the salvage pathway (e.g. sphinganine and dihydroceramides) (Figure S4A and S4B). In 4OHT-treated organoids, removing $\mathrm{C}_{2}$-ceramide from the media re-initiated the death program (Figure $3 \mathrm{~A}$ ).

To clarify which sphingolipid species might be important for organoid viability, we also exposed these organoid cultures to either the glucosylceramide synthase inhibitor D-threo-et-P4 or the sphingomyelin synthase inhibitor D609, which inhibit the production of glucosylceramides and sphingomyelin, respectively. Neither of these compounds recapitulated the myriocin effects on organoid survival (Figure S4C). Collectively, these data suggest that intermediates in the de novo sphingolipid synthesis pathway (e.g. sphingosine, ceramides or phytoceramides), rather than the abundant complex sphingolipids that comprise the majority of the sphingolipidome, (e.g. sphingomyelins), were requisite for organoid viability.

To confirm that $\mathrm{C}_{2}$-ceramide modulated stemness, we quantified the number of crypt domains per organoid following exposure to $\mathrm{C}_{2}$-ceramide. As we anticipated, treating the secondary organoids with $\mathrm{C}_{2}$-ceramide decreased the number of buds sprouting from each organoid, supporting the idea that sphingolipids enhanced stemness (Figure 3B).

\section{Ceramides enhance stemness by stimulating FABP1 expression to increase fatty acid uptake}

To explore the molecular mechanism(s) that might account for the profound sphingolipid actions in the intestinal epithelium, we conducted RNA sequencing on Sptlc2-knockout organoids treated with or without $\mathrm{C}_{2}$-ceramide. Fabpl, which encodes a fatty acid binding protein (i.e. FABP1) involved in lipid uptake, was amongst the handful of transcripts most dramatically upregulated by $\mathrm{C}_{2}$-ceramide (Figure $3 \mathrm{C}$ ). This $\mathrm{C}_{2}$-ceramide effect on $\mathrm{FABP} 1$ was particularly interesting owing to a bevy of studies showing that saturated fatty acids increase the expression of FABP1 in ISCs (Mihaylova et al., 2018) and that increasing fatty acid oxidation enhances stemness (Bensard et al., 2019; Knobloch et al., 2017; Mihaylova et al., 2018). Sptlc2 depletion from Sptlc2 $2^{\text {SEC }}$ mice decreased Fabpl transcripts in the crypt, but not the villi (Figure 3D). Using organoids, we also confirmed that Sptlc2 depletion decreased, and $\mathrm{C}_{2}$-ceramide restored, expression of Fabpl mRNA and FABP1 protein (Figure 3E and 3F).

To evaluate the functional consequences of these interventions on lipid handling, we quantified fatty acid uptake in organoids isolated from the $S p t l c 2^{\delta I E C}$ mice treated with vehicle, $4 \mathrm{OHT}$, or $\mathrm{C}_{2}$-ceramide. $\mathrm{C}_{2}$-ceramide stimulated fatty acid uptake in organoids. By contrast, depletion of Sptlc2 using 4OHT reduced rates of fatty acid uptake (Figure 3G).

The RNA sequencing also identified the transcript encoding carnitine palmitoyltransferase1 (Cptla), the enzyme that facilitates fatty acid entry into mitochondria, as a $\mathrm{C}_{2}$-ceramideresponsive gene. We confirmed that $\mathrm{C}_{2}$-ceramide slightly increased expression of Cpt $1 \mathrm{a}$ transcripts (by qPCR, Figure 4A) and CPT1a protein (by Western blot, Figure 4B). The CPT1 inhibitor etomoxir negated the protective actions of $\mathrm{C}_{2}$-ceramide on survival of Sptlc2 knockout organoids (Figure $3 \mathrm{H}$ ). Thus, induction of fatty acid uptake and utilization were essential $\mathrm{C}_{2}$-ceramide actions that preserved ISC viability.

\section{Ceramides increase FABP1 expression through PPAR $\alpha$ activation}

Prior studies have shown that the transcription factors peroxisome-proliferator activated receptor isoforms alpha $(\operatorname{PPAR} \alpha)$ and delta (PPAR $\delta)$ regulate FABP1 expression in the small 
intestine (Darimont et al., 1998; Hughes et al., 2015; Mochizuki et al., 2001; Poirier et al., 2001). In addition, recent studies have implicated PPAR $\delta$ as a critical intermediate linking saturated fats to the control of fatty acid oxidation and the enhancement of ISC stemness (Beyaz et al., 2016; Mihaylova et al., 2018). We thus tested whether Fabpl or Cpt1 expression was controlled by either of these transcription factors. The PPAR $\alpha$ antagonist GW6471 blocked the $\mathrm{C}_{2}$-ceramide induction of Fabpl (Figure 4A and 4B), but not Cpt1a. The PPAR $\delta$ antagonist GSK 3787 had no effect on either gene (Figure 4A). Thus, PPAR $\alpha$, but not PPAR $\delta$, was an essential intermediate linking $\mathrm{C}_{2}$ ceramide to the induction of FABP1.

Lastly, we evaluated whether PPAR $\alpha$ was sufficient to maintain organoid viability, even in the absence of Sptlc2. The PPAR $\alpha$ agonist GW7647 restored viability of organoids lacking Sptlc2 (Figure 4C and 4D). GW7647-treated organoids had fewer crypts in each organoid, thus recapitulating the $\mathrm{C}_{2}$-ceramide effect on organoid stemness (Figure 4E and 4F). Moreover, PPAR $\alpha$ activation recapitulated the ceramide effect on Fabpl expression (Figure 4G). These studies convincingly display the existence of a sphingolipid-PPAR $\alpha-F A B P 1$ axis that links macronutrients to the maintenance of the ISC pool.

\section{Discussion}

These studies provide important mechanistic insight about the nutritional signals that control the metabolism and proliferation of ISCs, the cell-of-origin of gastrointestinal cancers. Prior studies have shown that the transition from normal ISCs into a hyperplastic lesion is dependent upon fuel choice (Bensard et al., 2020). Excessive fatty acid supply increases the proliferation and stemness of ISC and progenitors within the crypt to promote tumorigenicity (Bensard et al., 2019; Knobloch et al., 2017; Mihaylova et al., 2018). In mouse models of colorectal cancer, genetic depletion or pharmacological inhibition of the mitochondrial pyruvate carrier, which increases dependence on fatty acids oxidation, expands the stem cell compartment and doubles the frequency of adenoma formation (Bensard et al., 2020; Schell et al., 2017). By contrast, germline deletion of Fabpl slows lipid uptake and reduces adenoma formation (Dharmarajan et al., 2013). The findings described herein reveal that sphingolipids produced from ectopic palmitate and serine influence this fuel choice by enhancing fatty acid import through FABP1.

Our data further identify PPAR $\alpha$ as an essential intermediate that links endogenous sphingolipids to the induction of Fabpl. This is consistent with prior studies, which show that high fat diet increases levels of Ppara and its downstream target Fabpl in rat jejunum (Suruga et al., 1999), as well as those showing that a PPAR $\alpha$ antagonist induces organoid death (Stine et al., 2019). Moreover, it aligns with studies showing that sphingolipids increase PPAR $\alpha$-mediated transcription (Correnti et al., 2018; Murakami et al., 2011; Tsuji et al., 2009; Van Veldhoven et al., 2000). Ceramides, phytoceramides, and sphingoid bases have been identified as upstream activators of PPAR $\alpha$, with some synthetic sphingolipids showing direct binding capabilities (Murakami et al., 2011; Tsuji et al., 2009; Van Veldhoven et al., 2000). Our findings reveal that one or more of these sphingolipids serves as a signal to increase fatty acid uptake and oxidation, and thus provides important new information about the nutritional sensing machinery that induces this transcriptional program.

The studies are also important for discerning the precise role of SPT in the gut. Prior studies have shown that genetic ablation of Sptlc2 (Li et al., 2018; Ohta et al., 2009) or pharmacological inhibition of SPT (Genin et al., 2016) has deleterious consequences on gut health, with the former causing animal death and the latter gastric enteropathy. Indeed, inflammatory bowel disease has been associated with decreased expression of SPT (Li et al., 2018). The studies described herein 
indicate that these pathogenic states result from decreased conversion of free fatty acid and amino acids into ceramides and a concomitant depletion of the ISC pool. Thus, strategies targeting sphingolipids could prove effective as a means of modulating the health of the intestinal barrier.

Collectively, these data support the conclusion that overproduction of sphingolipids enhances stemness of cells within the intestinal crypts. Using the organoid system, we identified an intriguing mechanism linking sphingolipids to the modulation of stemness through their effects on PPAR $\alpha$-mediated induction of FABP1 and a resultant increase in fatty acid uptake. These observations help reveal how saturated fatty acids control intestinal stemness and provide an explanation for the utility of serine deprivation as a means to decrease tumor incidence. Finally, because stem cells play a major role not only in tissue regeneration but also in carcinogenesis, deciphering their response to dietary factors is of the utmost importance for the treatment of degenerative conditions. Ultimately, these studies could suggest dietary or pharmacological strategies for maximizing regenerative capacity while also minimizing the risk of cancer development.

\section{Author contributions}

S.A.S, B.C., and Y.L. conceived of the project designed the experiments and wrote the manuscript. Y.L, B.C, V.A.K., J.A.M., performed experiments and analyzed data. C.B, J.R, H.C, Y.H provided some of the mouse strains used in this study. J.L.W and W.L.H. assisted in quantification of images. D.L provided human tissues used in this study and J.C, P.W and P.J.M aided in lipid analysis.

\section{Acknowledgements}

We wish to acknowledge the support from Metabolomics, Histology, and Metabolic Phenotyping Cores at the Health Sciences Center of the University of Utah. All data are available in the main text or the supplementary materials. Funding: Mass spectrometry equipment for the Metabolomics core was obtained through NCRR shared instrumentation grants 1S10OD016232-01, 1S10OD018210-01A1 and 1S10OD021505-01 and microscopy equipment for histology was obtained using a NCRR Shared Equipment Grant \# 1S10RR024761-01. Preclinical Research Resource was supported by the National Cancer Institute of the National Institutes of Health under Award Number P30CA042014. The authors received research support from the National Institutes of Health (DK115824, DK116888, and DK116450 to SAS; DK124326 to B.C; and DK108833 and DK112826 to WLH), the Juvenile Diabetes Research Foundation (JDRF 3-SRA-2019-768-AB to SAS and JDRF 3-SRA-2019-768-A-B to WLH), the American Diabetes Association (to SAS), the American Heart Association (to SAS), the Margolis Foundation (to SAS), and the United States Department of Agriculture (2019-67018-29250 to B.C.). J.L.W. received support by the National Institutes of Health through the Ruth L. Kirschstein National Research Service Award 5T32DK091317 from the National Institute of Diabetes and Digestive and Kidney Diseases.

\section{Declaration of Interests.}

SAS is a founder and shareholder with Centaurus Therapeutics.

Figure 1. Sphingolipids and sphingolipid-synthesizing genes are upregulated in human colon cancer. (A) Rough schematic of the sphingolipid synthesis pathway. Shown in bold font are the lipids, and in red the transcripts, that were upregulated in human colon cancer (see $\mathrm{C}$ and D, below). (B) Major lipid species in the mouse small intestine. Sphingolipids are in light blue. 
(C) Quantification of sphingolipids in colon cancer tissue and adjacent non-tumorous tissue from patients. (D) qPCR analysis of transcripts encoding sphingolipid synthesizing enzymes in colon cancer tissue or adjacent non-tumorous tissue obtained from patients. (E) Triacylglycerol and diacylglycerol in colon cancer tissue and adjacent non-tumorous tissue obtained from patients. $\left({ }^{*} \mathrm{p}<0.05,{ }^{* *} \mathrm{p}<0.01,{ }^{* *} \mathrm{p}<0.001, \mathrm{n}=9\right)$. Abbreviations: PE, phosphatidylethanolamine; PI, phosphatidylinositol; DAG, diacylglycerol; PC, phosphatidylcholine; Cho, cholesterol; TAG, triacylglycerol; CE, cholesterol esthers; SM, sphingomyelin; LPC, lysophosphatidylcholine; Cer, ceramide; PS, phosphatidylserine; Dh-Cer, dihydroceramide; Mhc-Cer, monohexosylceramide; Sptlc1, serine palmitoyltransferase long chain base subunit 1; Sptlc2, serine palmitoyltransferase, long chain base subunit 2; Cers6, ceramide synthase-6; Degs 1, dihydroceramide desaturase-1; Degs 2, dihydroceramide desaturase-2; Sgms1, sphingomyelin synthase-1; Sgms2, sphingomyelin synthase-2.

Fig. 2. Deletion of Sptlc2 from intestines disrupts the epithelium and depletes intestinal stem cells. $[A-H] S p t l c 2^{\delta I E C}$ and Sptlc $2^{f l f l}$ mice were given tamoxifen by intraperitoneal injection (1mg/day for 3 consecutive days). (A) Measurement of Sptcl2 levels in jejunum and colon 2-days after they received the first tamoxifen dose. (B) Body weight of Sptlc2 $2^{\delta I E C}$ and Sptlc ${ }^{f l f l}$ littermates assessed daily after tamoxifen injection. (C) Image of small intestine collected 4 days after receiving the first tamoxifen dose. (D) Two days after administering the first tamoxifen dose, FITC-dextran was given by oral gavage. Shown are serum levels 4-hours later. (E) H\&E staining of the jejunum 4-days after tamoxifen administration. (F) Histology of the jejunum 4-days after tamoxifen including periodic acid-Schiff (PAS) staining for goblet cells, LYZ1 immunostaining for Paneth cells, OLFM4 staining for ISC, and Ki67 staining to identify proliferating cells. (G) Time course study showing OLFM4 positive cells in tissues collected each day after tamoxifen administration. (H) Quantification of crypts per villus from Sptlc2 $2^{\delta I E C}$ and $S p t l c^{f l f l}$ mice. [I-J] Sptlc $2^{\delta I S C}$ and Sptlc $2^{\text {flflfl }}$ mice were given tamoxifen by intraperitoneal injection (3mg/day for 5 consecutive days). (I) In situ hybridization (RNAscope) measuring Sptlc2, Olfm4, and Lyz1 mRNA expression in the intestine 5 days after tamoxifen injection. Nuclei were stained by DAPI. (J) H\&E staining of epithelium from jejunum 5 days after the first tamoxifen dose. Abbreviations: IEC, intestinal epithelia cell; PC, phosphatidylcholine; FITC-Dextran, Fluorescein isothiocyanatedextran; H\&E, Hematoxylin and Eosin staining; PAS, periodic acid-Schiff; Lyz1, Lysozyme C1; Olfm4, Olfactomedin 4; ISC, intestinal stem cell; serine palmitoyltransferase, long chain base subunit 2; DAPI, 4',6-diamidino-2-phenylindole.

Fig. 3. Ceramides promote stemness and intestinal organoid survival by stimulating FABP1 expression to enhance fatty acid uptake. Intestinal organoids were cultured from crypts isolated from the jejunum of Sptlc $c^{\delta I E C}$ mice. (A) (left) Propidium iodide (PI) staining of organoids treated with either vehicle, myriocin $(10 \mu \mathrm{M})$, or $4 \mathrm{OHT}(200 \mathrm{ng} / \mathrm{ml})$ for 48 hours. Some samples were supplemented with $\mathrm{C}_{2}$-ceramide $\left(25 \mu \mathrm{M}\right.$, Cer). In the sample on the lower right, the added $\mathrm{C}_{2}$ ceramide was then removed from the media (washout) for the final 24 hours. (right) Quantification of the organoid staining reported as relative fluorescence units (RFU) normalized to the area of the organoid. (B) Secondary organoids treated with either vehicle or $\mathrm{C}_{2}$-ceramide $(25 \mu \mathrm{M})$ for 7 days. On the right is a quantification of the crypts growing from each organoid. $(n=50, * p<0.05$, $* * \mathrm{p}<0.01, * * * \mathrm{p}<0.001)$. (C) Volcano plot depicting the RNAseq datasets obtained from 4OHTtreated organoids $(200 \mathrm{ng} / \mathrm{ml})$ supplemented with or without $\mathrm{C}_{2}$-ceramide $(25 \mu \mathrm{M})$ for 48 hours. (D) qPCR analysis of Fabpl expression in the villi or crypts from the jejuna of the Sptlc $c^{f l f l}$ or 
Sptlc ${ }^{\delta I E C}$ mice. (E) qPCR analysis of Fabpl gene expression in organoids treated with vehicle, 4OHT $(200 \mathrm{ng} / \mathrm{ml})$, or $4 \mathrm{OHT}+\mathrm{C}_{2}$-ceramide $(\mathrm{Cer}, 25 \mu \mathrm{M})$ for 48 hours. (F) Western blot showing FABP1 and actin expression following Sptlc2 deletion with 4-OHT and/or $\mathrm{C}_{2}$-ceramide supplementation for 48 hours (quantification on the right). (G) Fatty acid uptake into cells dissociated from organoids and treated with vehicle, $4 \mathrm{OHT}$, and/or $\mathrm{C}_{2}$-ceramide for 48 hours. The bar graph depicts the area under the curve. $(\mathrm{H})$ (left) PI staining images of organoid treated with vehicle, 4OHT, 4OHT plus C2-ceramide or 4OHT plus C2 ceramide plus etomoxir $(100 \mu \mathrm{M})$. (right) Quantification of PI staining analyzed by imageJ software $\left(n=30,{ }^{*} p<0.05, * * p<0.01\right.$, $* * * \mathrm{p}<0.001)$. Abbreviations: Cer, (d18:1/2:0) N-acetoyl-D-erythro-sphingosine; 4-OHT, 4hydroxytamoxifen; RFU, relative fluorescence unit; IEC, intestinal epithelia cell; FABP1, fatty acid binding protein 1; AUC, area under the curve; Sptlc2, serine palmitoyltransferase; Cpt1a, Carnitine palmitoyltransferase $1 \mathrm{~A}$.

Fig. 4. Ceramides increases FABP1 expression through PPAR $\alpha$ activation. (A) qPCR analysis of Fabpl and Cpt la expression in organoids treated with vehicle, C2-ceramide $(25 \mu \mathrm{M})$, and $\mathrm{C}_{2}-$ ceramide with GW6471(PPAR $\alpha$ antagonist, $1 \mu \mathrm{M}$ ) or GSK3787 (PPAR $\delta$ antagonist, $1 \mu \mathrm{M}$ ) for 48 hours. (B) Western blot analysis of FABP1, CPT1a, and actin in organoids treated with $\mathrm{C}_{2-}$ ceramide with or without the PPAR $\alpha$ antagonist GW6471(GW6471, $1 \mu \mathrm{M})$ for 48 hours. (C) Propidium iodide (PI) staining of organoids treated with either vehicle, 4OHT $(200 \mathrm{ng} / \mathrm{ml})$ or 4OHT with the PPAR $\alpha$ agonist GW7647 for 48 hours. (D) Quantification of PI staining of 4C. (E) Secondary organoids treated with either vehicle or GW7647 $(1 \mu \mathrm{M})$ for 7 days. (F) Quantification of the crypts growing from each organoid. ( $\mathrm{n}=50, * \mathrm{p}<0.05, * * \mathrm{p}<0.01, * * * \mathrm{p}<0.001)$. (G) $\mathrm{qPCR}$ analysis of Fabpl expression in organoids treated with either vehicle or GW7647 $(1 \mu \mathrm{M})$. $(\mathrm{H})$ Schematic depicting the concept shown herein that SPT-generated sphingolipids are obligate intermediates linking saturated fatty acids and serine to stemness. The picture is generated using Biorender software (Biorender.com). Abbreviations: Cer, (d18:1/2:0) N-acetoyl-D-erythrosphingosine; 4-OHT, 4-hydroxytamoxifen; RFU, relative fluorescence unit; IEC, intestinal epithelia cell; FABP1, fatty acid binding protein 1; Cpt1a, Carnitine palmitoyltransferase 1A; PPAR $\alpha$, Peroxisome proliferator-activated receptor alpha; AUC, area under the curve; FAO, fatty acid oxidation.

Table 1: Patient information

\begin{tabular}{|l|l|l|l|l|l|l|l|}
\hline & Gender & Age & BMI & $\begin{array}{l}\text { Hemoglobin } \\
(\mathrm{g} / \mathrm{dl})\end{array}$ & $\begin{array}{l}\text { Primary Tumor } \\
(\text { tumor depth) }\end{array}$ & $\begin{array}{l}\text { Histologic } \\
\text { Type }\end{array}$ & Histologic Grade \\
\hline Mean & & 68.56 & 26.33 & 11.88 & & Ad & \\
\hline Range & & $50-84$ & $21.3-34.4$ & $7.6-15.1$ & & Ad & \\
\hline 1 & M & 61 & 34.4 & 12.9 & pT3 & Ad & Moderately Diff. \\
\hline 2 & M & 84 & 23.3 & 15.1 & pT2 & Ad & Well to Moderately Diff. \\
\hline 3 & M & 81 & 23.25 & 14.6 & pT3 & Ad & Moderately Diff. \\
\hline 4 & M & 50 & 30.3 & 10.8 & pT3 & Ad & Moderately Diff. \\
\hline 5 & F & 50 & 22.9 & 10.3 & pT2 & Ad & Well Diff. \\
\hline 6 & F & 58 & 27.6 & 10.7 & pT4a & Ad & Well Diff. \\
\hline 7 & F & 80 & 21.3 & 12.3 & pT2 & Ad & Moderately Diff. \\
\hline 8 & F & 74 & 30.4 & 12.6 & pT3 & Ad & Well Diff. \\
\hline 9 & M & 79 & 23.5 & 7.6 & pT3 & Ad & Moderately Diff. \\
\hline
\end{tabular}

Ad: Adenocarcinoma. 


\section{Materials and Methods}

\section{Human colon samples}

Human colon cancer tissues were obtained from the Preclinical Research Resource at the Huntsman Cancer Institute. Tumors were collected during tumor resection (not biopsies) and cryopreserved as viable tissue. The adjacent normal tissue was collected as a paired control.

\section{Animal Colonies}

All animal experiments were conducted with protocols approved by the Institutional Animal Care and Use Committee (IAVUC) of the University of Utah. Generation of Sptlc2 ${ }^{\delta I E C}$ Mice. Sptlc2 $2^{f l / f l}$ mice were crossed with Villin-Cre-ERT2 mice(Goh et al., 2015) to generate gut-specific Sptlc2 knockout animals. To deplete the gene, mice were injected intraperitoneally with tamoxifen $\left(1 \mathrm{mg} /\right.$ mouse) every day for three days at 8-10 weeks of age. Sptlc $2^{\text {flffl }}$ mice littermates that were also injected with tamoxifen were used as controls. Generation of Sptlc2 ${ }^{\delta I S C}$ Mice. Sptlc $2^{\text {fllfl }}$ mice were crossed with Olfm4-EGFP-ires-CreERT2 mice (26) to generate ISC-specific Sptlc2 knockout animals. To deplete the gene, mice were injected intraperitoneally with tamoxifen ( $3 \mathrm{mg} / \mathrm{mouse})$ every day for five days at 8-10 weeks of age. Sptlc $2^{f l f l}$ mice littermates that were also injected with tamoxifen were used as controls.

\section{Sphingolipidomics}

University of Utah Metabolomics Core: Tissues or organoids suspended in $120 \square 1$ PBS went through three freeze-thaw cycles to homogenize the samples. Thereafter, $100 \square 1$ of homogenate was used for lipid extraction and $20 \square 1$ was saved for protein assays. Samples were spiked with $225 \mu \mathrm{L} \mathrm{MeOH}$ containing internal standards (deuterated ceramide mixture (C16, $60 \mathrm{pmol} / \mathrm{sample}$; C18, $35 \mathrm{pmol} / \mathrm{sample}$; $24,150 \mathrm{pmol} / \mathrm{sample}$; $24: 1,312 \mathrm{pmol} / \mathrm{sample})$; dihydroceramide C18:1, $5 \mathrm{pmol} / \mathrm{sample}$; deuterated sphingomyelin, $50 \mathrm{pmol} / \mathrm{sample}$; glucosylceramide $\mathrm{C} 17,50$ $\mathrm{pmol} / \mathrm{sample}$; and deuterated S1P, 5pmol/sample) followed by $750 \mu \mathrm{L}$ MTBE (methyl tert-butyl ether). The samples were then incubated for $60 \mathrm{~min}$ on ice with brief vortexing every $15 \mathrm{~min}$. Following centrifugation for phase separation, the upper phase was transferred to another Eppendorf tube which was dried using a Speedvac. The dried, extracted lipid was reconstituted in methanol:toluene (9:1). The lipid extracts were then analyzed by liquid chromatography-mass spectrometry (LC/MS). Briefly, lipid extracts were separated on an Acquity UPLC CSH C18 1.7 $\mu \mathrm{m} 2.1 \times 50 \mathrm{~mm}$ column maintained at $60^{\circ} \mathrm{C}$ connected to an Agilent HiP 1290 Sampler, Agilent 1290 Infinity pump, and Agilent 6490 triple quadrupole (QqQ) mass spectrometer. Sphingolipids were detected using dynamic multiple reaction monitoring (dMRM) in positive ion mode. Source gas temperature was set to $210^{\circ} \mathrm{C}$, with a gas $\left(\mathrm{N}_{2}\right)$ flow of $11 \mathrm{~L} / \mathrm{min}$ and a nebulizer pressure of 30 psi. Sheath gas temperature was $400^{\circ} \mathrm{C}$, sheath gas $\left(\mathrm{N}_{2}\right)$ flow of $12 \mathrm{~L} / \mathrm{min}$, capillary voltage is 4000 $\mathrm{V}$, nozzle voltage $500 \mathrm{~V}$, high pressure RF $190 \mathrm{~V}$ and low-pressure RF is $120 \mathrm{~V}$. Injection volume was $2 \mu \mathrm{L}$ and the samples were analyzed in a randomized order with the pooled QC sample injection eight times throughout the sample queue. Mobile phase A consisted of $\mathrm{ACN}: \mathrm{H}_{2} \mathrm{O}(60: 40$ $\mathrm{v} / \mathrm{v})$ and mobile phase B consists of IPA:ACN: $\mathrm{H}_{2} \mathrm{O}(90: 9: 1 \mathrm{v} / \mathrm{v})$ both in $10 \mathrm{mM}$ ammonium formate and $0.1 \%$ formic acid. The chromatography gradient started at $15 \%$ mobile phase $\mathrm{B}$, increased to $30 \% \mathrm{~B}$ over $1 \mathrm{~min}$, increased to $60 \% \mathrm{~B}$ from $1-2 \mathrm{~min}$, increased to $80 \% \mathrm{~B}$ from $2-10$ min, and increased to $99 \%$ B from 10-10.2 min where it was held until 14 min. Post-time was 5 min and the flowrate was $0.35 \mathrm{~mL} / \mathrm{min}$ throughout. Collision energies and cell accelerator voltages were optimized using sphingolipid standards with dMRM transitions as $[\mathrm{M}+\mathrm{H}]^{+} \rightarrow[\mathrm{m} / \mathrm{z}=284.3]$ 
for dihydroceramides, $[\mathrm{M}+\mathrm{H}]^{+} \rightarrow[\mathrm{m} / \mathrm{z}=287.3]$ for isotope labeled dihydroceramides, [M$\left.\mathrm{H}_{2} \mathrm{O}+\mathrm{H}\right]+\rightarrow[m / z=264.2]$ for ceramides, $\left[\mathrm{M}-\mathrm{H}_{2} \mathrm{O}+\mathrm{H}\right]^{+} \rightarrow[m / z=267.2]$ for isotope labeled ceramides and $[\mathrm{M}+\mathrm{H}]^{+} \rightarrow\left[\mathrm{M}-\mathrm{H}_{2} \mathrm{O}+\mathrm{H}\right]+$ for all targets. Sphingolipids without available standards were identified based on HR-LC/MS, quasi-molecular ion and characteristic product ions. Their retention times were either taken from HR-LC/MS data or inferred from the available sphingolipid standards. Results from LC/MS experiments are collected using Agilent Mass Hunter Workstation and analyzed using the software package Agilent Mass Hunter Quant B.07.00. Sphingolipids were quantitated based on peak area ratios to the standards added to the extracts.

Baker Heart and Diabetes Institute Metabolomics Laboratory: The quantification of specific lipids at the Baker Heart and Diabetes Institute Metabolomics laboratory was done as follows: $200 \mu \mathrm{L}$ of chloroform/methanol (2:1, v/v) were added together with $10 \mu \mathrm{L}$ ISTD in chloroform/methanol $(1: 1, \mathrm{v} / \mathrm{v})$ to $25-60 \mu \mathrm{g}$ of protein lysate in $10 \mu \mathrm{l}$ cold PBS. The mixture was mixed for $10 \mathrm{~min}$ on a rotary mixer, sonicated in a water bath $\left(18{ }^{\circ} \mathrm{C}-24^{\circ} \mathrm{C}\right)$ for $30 \mathrm{~min}$, left to stand on the bench for 20 min and then centrifuged $\left(16,000 \times \mathrm{g}, 10 \mathrm{~min}, 20^{\circ} \mathrm{C}\right)$. The supernatant was transferred to a $96-$ well plate and dried under a stream of nitrogen gas at $40{ }^{\circ} \mathrm{C}$. Samples were reconstituted with 50 $\mu \mathrm{L} \mathrm{H}_{2} \mathrm{O}$-saturated 1-butanol and sonicated for $10 \mathrm{~min}$. Finally, $50 \mu \mathrm{L}$ of $10 \mathrm{mM}$ ammonium formate in $\mathrm{MeOH}$ was added. The extract was centrifuged $\left(1700 \times \mathrm{g}, 5 \mathrm{~min}, 20{ }^{\circ} \mathrm{C}\right)$ and the supernatant was transferred into a $0.2 \mathrm{~mL}$ glass insert with Teflon insert cap for analysis by LC ESI-MS/MS as described (27).

Isolation, culture, and analysis of intestinal organoids

Small intestinal crypts were isolated and grown into organoids as described previously(Sato et al., 2009). Isolated small intestines were chopped into small pieces and washed in cold PBS. They were then incubated in $2 \mathrm{mM}$ EDTA with PBS for $30 \mathrm{~min}$ on ice. EDTA medium was removed and the tissue fragments were washed by cold PBS. They were then pipetted vigorously to dissociate and enrich the crypts into the supernatant. Isolated crypts were pull down by centrifuged at 200g, and then re-suspended in Matrigel (Car\#354277, BD) for organoids culture. Myriocin $(10 \mathrm{uM})$ was used to inhibit SPT2 activity in the organoids for 24-48 hours. Deletion of Sptlc2 in organoid cultures from Sptlc2 ${ }^{\square I E C}$ mice was induced by addition of 200ng/ml 4-OH-tamoxifen (4OHT) to the culture medium for 48 hours. Cell death of organoid cultures was determined by propidium iodine (PI)-positive staining. Images were taken by EVOS digital inverted microscope. Quantification of PI staining was done by Image J software (NIH).

Antibiotic treatment of Sptlc2 $2^{\delta I E C}$ mice

A broad-spectrum antibiotic mixture was added to the drinking water one month before the ablation of the Sptlc2 gene(Takahashi et al., 2014). The cocktail included 200mg ciprofloxacin (Sigma-Aldrich), 1g ampicillin (Sigma-Aldrich), 100mg metronidazole (Sigma-Aldrich), 500mg vancomycin (Labconsult), 1g neomycin, 2.50g streptomycin (ThermoFisher), 100,000U penicillin (ThermoFisher), $1 \mathrm{~g}$ bacitracin (Sigma-Aldrich) and $1 \mathrm{~g}$ ceftazidime (Sigma-Aldrich) were dissolved in 1 liter of water. Feces was collected and cultured in liquid cultures of Thioglycollate medium (Sigma) and McConkey agar plates. No bacteria growth was observed from both cultures.

Quantitative RT-PCR

Total RNA was extracted from tissues or cells using the RNeasy Mini Isolation Kits with DNA digestion (QIAGEN) according to the manufacturer's instructions. Isolated total RNA was reverse- 
transcribed into cDNA using commercially available kits (Quantbio). All subsequent qRT-PCR reactions were performed on a QuantStudio 12K Flex Real-Time PCR system (Thermo Fisher Scientific) using the Qiagen QuantiFast SYBR Green PCR kit. qPCR primers were purchased from Sigma-Aldrich, KiCqStart Primers. For normalization threshold cycles ( $\mathrm{C}_{\mathrm{t}}$-values) of all replicate analyses were normalized to Ppib or B2M for mouse tissues and human tissue within each sample to obtain sample-specific $\Delta \mathrm{C}_{\mathrm{t}}$ values $\left(=\mathrm{C}_{\mathrm{t}}\right.$ gene of interest $-\mathrm{C}_{\mathrm{t}}$ Ppib, B2M). To compare the effect of various treatments with untreated controls, $2^{-\Delta \Delta \mathrm{Ct}}$ values were calculated to obtain fold expression levels, where $\Delta \Delta \mathrm{C}_{\mathrm{t}}=\left(\Delta \mathrm{C}_{\mathrm{t}}\right.$ treatment $-\Delta \mathrm{C}_{\mathrm{t}}$ control $)$.

\section{FITC-Dextran Permeability Test}

Intestinal permeability was assessed by oral gavage of FITC-dextran (Sigma-Aldrich). Mice were administered $500 \square 1$ FITC-Dextran $(20 \mathrm{mg} / \mathrm{ml}$ in PBS, $10 \mathrm{mg} / \mathrm{mouse}$ ) by gavage. Serum sample were collected 4-hours after oral gavage; Fluorescence were measured by a fluorescence plate reader at $488 \mathrm{~nm}$. Serum FITC-Dextran concentration was calculated according to the standard curve made by a serial dilution from the stock in PBS.

\section{$\underline{\text { RNAseq Analysis. }}$}

Total RNA was extracted and RNAseq analysis was conducted at University of Utah genomics core facility who evaluated the RNA quality by NanoDrop spectrophotometer and Agilent Bioanalyzer RNA 6000 Chip and further performed the sequencing by Illumina HiSeq2000 with 50 cycle paired end sequencing. Data were analyzed by bioinformatics analysis service at the microarray core facility at the University of Utah. The criteria for differential expression included only genes with FDR of $<0.05$ or log-transformed false discovery rate (FDR) of $>13$ and with a fold change greater than or equal to \pm 1.5 relative to controls. The Fragments Per Kilobase Million (FPKM) values of the differentially expressed genes were $\log$ transformed and normalized to the average of the controls. These values were used to produce hierarchical clustering and generate heat maps of the genes using Cluster 3.0 and Java Tree View open source programs, respectfully.

\section{Western Blot Analysis}

Proteins were extracted from organoids by homogenizing in RIPA buffer $(0.5 \%$ NP-40, $0.1 \%$ sodium deoxycholate, $150 \mathrm{mM} \mathrm{NaCl}, 50 \mathrm{mM}$ Tris-HCl, $\mathrm{pH}$ 7.5) containing protease inhibitors (Complete Mini, Roche). The homogenate was cleared by centrifugation at $4{ }^{\circ} \mathrm{C}$ for $30 \mathrm{~min}$ at $15,000 \mathrm{~g}$ and the supernatant containing the protein fraction recovered. Protein concentration in the supernatant was determined using the BCA Protein Assay Kit (Pierce). $10 \mu \mathrm{g}$ of proteins was resolved using precast Bolt (4-12\% Bis-Tris gels, Invitrogen) and transferred to Nitrocellulose membranes (GE Healthcare). Membranes were blocked with 5\% BSA in Tris-buffered saline containing $0.2 \%$ Tween-20 (TBS-T) and incubated with primary antibodies at $4^{\circ} \mathrm{C}$ overnight.

\section{Histology and Immunochemistry}

For histology, tissues were fixed in $10 \%$ buffered formalin for 48 hours, embedded in paraffin, sectioned at $5 \mu \mathrm{m}$ and mounted on the slides. The slides were either stained with hematoxylin and eosin or PAS for goblet cells. Immunohistochemistry: The slides were sent to Arup laboratories for immunohistochemical analysis of Olfm4, Lyz1, Ki67, F4/80 and cleaved caspase-3 staining. 


\section{Fatty Acid Uptake Assay}

Organoids were grown and treated with vehicle, 4 OHT $(200 \mathrm{ng} / \mathrm{ml})$ or $\mathrm{C}_{2}$-ceramide for 48 hours. Organoids were then dissociated into single cell suspension and seeded onto a 384-well plate. BODIPY-dodecanoic acid fluorescent fatty acid analog was added (QBT ${ }^{\mathrm{TM}}$ Fatty Acid Uptake Assay Kit, Molecular Devices) and the plate was immediately transferred to a fluorescence microplate reader for kinetic reading (every 20 seconds for 30-60 minutes) using a bottom-read mode. Measurement was done with an excitation filter of $480 \mathrm{~nm}$ and an emission filter of 520 .

RNA fluorescence in situ hybridization

Formalin-fixed paraffin-embedded (FFPE) mouse jejunum sections were de-paraffinized, rehydrated, and then hybridized with mRNA probes against mouse Sptlc2, Olfm4 and Lyzl in accordance with the manufacturer's instructions (Advanced Cell Diagnostics, Newark, CA). In brief, FFPE sections were pretreated with hydrogen peroxide, incubated in Target Retrieval solution in a steamer for 30 minutes, and permeabilized by incubating in Protease Plus solution for 40 minutes. After hybridization, a fluorescent kit was used to amplify the mRNA signal. TSA Plus Fluorescein, TSA Plus Cyanine 3, and TSA Plus Cyanine 5 fluorescent signals were detected using EVOS digital inverted microscope.

\section{Statistical analysis}

Data were plotted as the mean \pm SEM. Student $t$-test, one-way or two-way ANOVA were carried out using Excel or prism and statistical significance was considered meaningful at $\mathrm{p}<0.05$.

\section{References and Notes}

Bensard, C.L., Wisidagama, D.R., Olson, K.A., Berg, J.A., Krah, N.M., Schell, J.C., Nowinski, S.M., Fogarty, S., Bott, A.J., Wei, P., et al. (2019). Regulation of Tumor Initiation by the Mitochondrial Pyruvate Carrier. Cell Metab.

Bensard, C.L., Wisidagama, D.R., Olson, K.A., Berg, J.A., Krah, N.M., Schell, J.C., Nowinski, S.M., Fogarty, S., Bott, A.J., Wei, P., et al. (2020). Regulation of Tumor Initiation by the Mitochondrial Pyruvate Carrier. Cell Metab 31, 284-300 e287.

Beyaz, S., Mana, M.D., Roper, J., Kedrin, D., Saadatpour, A., Hong, S.J., Bauer-Rowe, K.E., Xifaras, M.E., Akkad, A., Arias, E., et al. (2016). High-fat diet enhances stemness and tumorigenicity of intestinal progenitors. Nature 531, 53-58.

Chaurasia, B., Kaddai, V.A., Lancaster, G.I., Henstridge, D.C., Sriram, S., Galam, D.L., Gopalan, V., Prakash, K.N., Velan, S.S., Bulchand, S., et al. (2016). Adipocyte Ceramides Regulate Subcutaneous Adipose Browning, Inflammation, and Metabolism. Cell Metab 24, 820-834.

Correnti, J.M., Gottshall, L., Lin, A., Williams, B., Oranu, A., Beck, J., Chen, J., Bennett, M.J., and Carr, R.M. (2018). Ethanol and C2 ceramide activate fatty acid oxidation in human hepatoma cells. Sci Rep 8, 12923.

Darimont, C., Gradoux, N., Cumin, F., Baum, H.P., and De Pover, A. (1998). Differential regulation of intestinal and liver fatty acid-binding proteins in human intestinal cell line (Caco-2): role of collagen. Exp Cell Res 244, 441-447.

Dharmarajan, S., Newberry, E.P., Montenegro, G., Nalbantoglu, I., Davis, V.R., Clanahan, M.J., Blanc, V., Xie, Y., Luo, J., Fleshman, J.W., Jr., et al. (2013). Liver fatty acid-binding protein (L- 
Fabp) modifies intestinal fatty acid composition and adenoma formation in ApcMin/+ mice. Cancer Prev Res (Phila) 6, 1026-1037.

Genin, M.J., Gonzalez Valcarcel, I.C., Holloway, W.G., Lamar, J., Mosior, M., Hawkins, E., Estridge, T., Weidner, J., Seng, T., Yurek, D., et al. (2016). Imidazopyridine and Pyrazolopiperidine Derivatives as Novel Inhibitors of Serine Palmitoyl Transferase. J Med Chem 59, 5904-5910.

Goh, V.J., Tan, J.S., Tan, B.C., Seow, C., Ong, W.Y., Lim, Y.C., Sun, L., Ghosh, S., and Silver, D.L. (2015). Postnatal Deletion of Fat Storage-inducing Transmembrane Protein 2 (FIT2/FITM2) Causes Lethal Enteropathy. J Biol Chem 290, 25686-25699.

Hammerschmidt, P., Ostkotte, D., Nolte, H., Gerl, M.J., Jais, A., Brunner, H.L., Sprenger, H.G., Awazawa, M., Nicholls, H.T., Turpin-Nolan, S.M., et al. (2019). CerS6-Derived Sphingolipids Interact with Mff and Promote Mitochondrial Fragmentation in Obesity. Cell 177, 1536-1552 e1523.

Hughes, M.L., Liu, B., Halls, M.L., Wagstaff, K.M., Patil, R., Velkov, T., Jans, D.A., Bunnett, N.W., Scanlon, M.J., and Porter, C.J. (2015). Fatty Acid-binding Proteins 1 and 2 Differentially Modulate the Activation of Peroxisome Proliferator-activated Receptor alpha in a Ligand-selective Manner. J Biol Chem 290, 13895-13906.

Knobloch, M., Pilz, G.A., Ghesquiere, B., Kovacs, W.J., Wegleiter, T., Moore, D.L., Hruzova, M., Zamboni, N., Carmeliet, P., and Jessberger, S. (2017). A Fatty Acid Oxidation-Dependent Metabolic Shift Regulates Adult Neural Stem Cell Activity. Cell Rep 20, 2144-2155.

Leblond, C.P., and Stevens, C.E. (1948). The constant renewal of the intestinal epithelium in the albino rat. Anat Rec 100, 357-377.

Li, Z., Kabir, I., Tietelman, G., Huan, C., Fan, J., Worgall, T., and Jiang, X.C. (2018). Sphingolipid de novo biosynthesis is essential for intestine cell survival and barrier function. Cell Death Dis 9 , 173.

Maddocks, O.D.K., Athineos, D., Cheung, E.C., Lee, P., Zhang, T., van den Broek, N.J.F., Mackay, G.M., Labuschagne, C.F., Gay, D., Kruiswijk, F., et al. (2017). Modulating the therapeutic response of tumours to dietary serine and glycine starvation. Nature 544, 372-376.

Mihaylova, M.M., Cheng, C.W., Cao, A.Q., Tripathi, S., Mana, M.D., Bauer-Rowe, K.E., AbuRemaileh, M., Clavain, L., Erdemir, A., Lewis, C.A., et al. (2018). Fasting Activates Fatty Acid Oxidation to Enhance Intestinal Stem Cell Function during Homeostasis and Aging. Cell Stem Cell 22, 769-778 e764.

Mochizuki, K., Suruga, K., Yagi, E., Takase, S., and Goda, T. (2001). The expression of PPARassociated genes is modulated through postnatal development of PPAR subtypes in the small intestine. Biochim Biophys Acta 1531, 68-76.

Murakami, I., Wakasa, Y., Yamashita, S., Kurihara, T., Zama, K., Kobayashi, N., Mizutani, Y., Mitsutake, S., Shigyo, T., and Igarashi, Y. (2011). Phytoceramide and sphingoid bases derived from brewer's yeast Saccharomyces pastorianus activate peroxisome proliferator-activated receptors. Lipids Health Dis 10, 150. 
Ohta, E., Ohira, T., Matsue, K., Ikeda, Y., Fujii, K., Ohwaki, K., Osuka, S., Hirabayashi, Y., and Sasaki, M. (2009). Analysis of development of lesions in mice with serine palmitoyltransferase (SPT) deficiency -Sptlc2 conditional knockout mice. Exp Anim 58, 515-524.

Poirier, H., Niot, I., Monnot, M.C., Braissant, O., Meunier-Durmort, C., Costet, P., Pineau, T., Wahli, W., Willson, T.M., and Besnard, P. (2001). Differential involvement of peroxisomeproliferator-activated receptors alpha and delta in fibrate and fatty-acid-mediated inductions of the gene encoding liver fatty-acid-binding protein in the liver and the small intestine. Biochem $\mathrm{J} 355$, 481-488.

Raichur, S., Brunner, B., Bielohuby, M., Hansen, G., Pfenninger, A., Wang, B., Bruning, J.C., Larsen, P.J., and Tennagels, N. (2019). The role of C16:0 ceramide in the development of obesity and type 2 diabetes: CerS6 inhibition as a novel therapeutic approach. Mol Metab 21, 36-50.

Raichur, S., Wang, S.T., Chan, P.W., Li, Y., Ching, J., Chaurasia, B., Dogra, S., Ohman, M.K., Takeda, K., Sugii, S., et al. (2014). CerS2 Haploinsufficiency Inhibits beta-Oxidation and Confers Susceptibility to Diet-Induced Steatohepatitis and Insulin Resistance. Cell Metab 20, 919.

Sato, T., Vries, R.G., Snippert, H.J., van de Wetering, M., Barker, N., Stange, D.E., van Es, J.H., Abo, A., Kujala, P., Peters, P.J., et al. (2009). Single Lgr5 stem cells build crypt-villus structures in vitro without a mesenchymal niche. Nature 459, 262-265.

Schell, J.C., Wisidagama, D.R., Bensard, C., Zhao, H., Wei, P., Tanner, J., Flores, A., Mohlman, J., Sorensen, L.K., Earl, C.S., et al. (2017). Control of intestinal stem cell function and proliferation by mitochondrial pyruvate metabolism. Nat Cell Biol 19, 1027-1036.

Schuijers, J., van der Flier, L.G., van Es, J., and Clevers, H. (2014). Robust cre-mediated recombination in small intestinal stem cells utilizing the olfm4 locus. Stem Cell Reports 3, 234241.

Stine, R.R., Sakers, A.P., TeSlaa, T., Kissig, M., Stine, Z.E., Kwon, C.W., Cheng, L., Lim, H.W., Kaestner, K.H., Rabinowitz, J.D., et al. (2019). PRDM16 Maintains Homeostasis of the Intestinal Epithelium by Controlling Region-Specific Metabolism. Cell Stem Cell 25, 830-845 e838.

Summers, S.A., Chaurasia, B., and Holland, W.L. (2019). Metabolic Messengers: Ceramides. Nature Metabolism 1, 1051-1058

Suruga, K., Mochizuki, K., Kitagawa, M., Goda, T., Horie, N., Takeishi, K., and Takase, S. (1999). Transcriptional regulation of cellular retinol-binding protein, type II gene expression in small intestine by dietary fat. Arch Biochem Biophys 362, 159-166.

Takahashi, N., Vereecke, L., Bertrand, M.J., Duprez, L., Berger, S.B., Divert, T., Goncalves, A., Sze, M., Gilbert, B., Kourula, S., et al. (2014). RIPK1 ensures intestinal homeostasis by protecting the epithelium against apoptosis. Nature 513, 95-99.

Tsuji, K., Satoh, S., Mitsutake, S., Murakami, I., Park, J.J., Li, Q., Chang, Y.T., Chung, S.K., and Igarashi, Y. (2009). Evaluation of synthetic sphingolipid analogs as ligands for peroxisome proliferator-activated receptors. Bioorg Med Chem Lett 19, 1643-1646.

Turpin, S.M., Nicholls, H.T., Willmes, D.M., Mourier, A., Brodesser, S., Wunderlich, C.M., Mauer, J., Xu, E., Hammerschmidt, P., Bronneke, H.S., et al. (2014). Obesity-induced CerS6dependent $\mathrm{C} 16: 0$ ceramide production promotes weight gain and glucose intolerance. Cell Metab 20, 678-686. 
Van Veldhoven, P.P., Mannaerts, G.P., Declercq, P., and Baes, M. (2000). Do sphingoid bases interact with the peroxisome proliferator activated receptor alpha (PPAR-alpha)? Cell Signal 12, 475-479. 


\section{Figure 1}

A

Palmitoyl-CoA

bioRxiv preprint doi: https://toi.org/10.1101/2020.12.03.409128; this version posted December 4, 2020. The copyright holder for this preprint

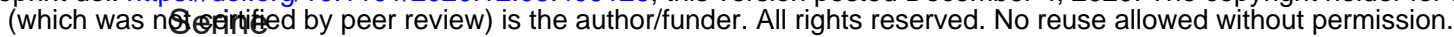

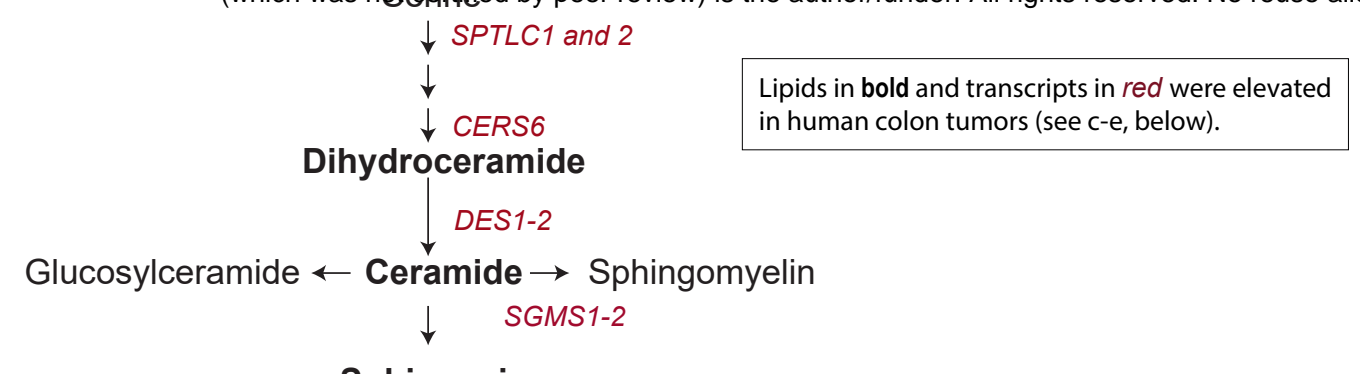

\section{Sphingosine}

B

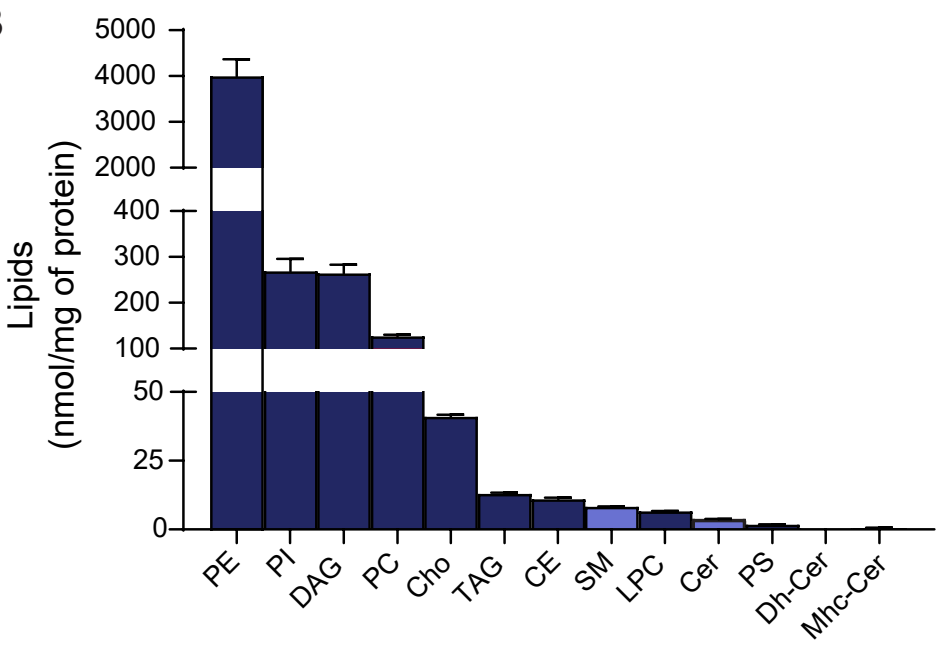

C

Dihydroceramides

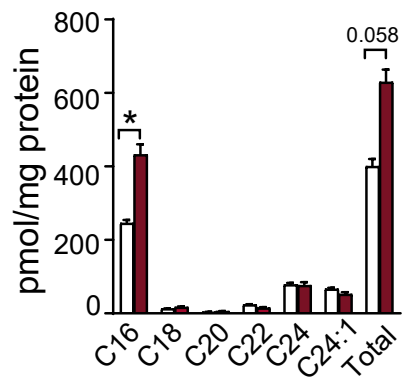

D

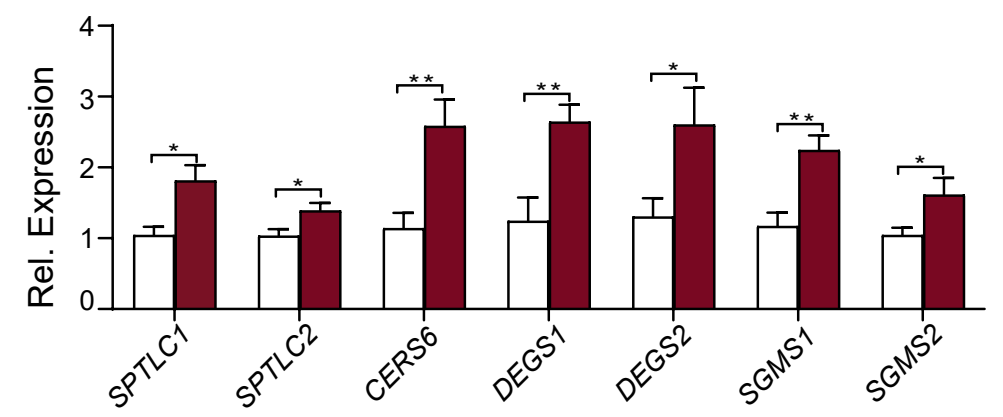

Ceramides

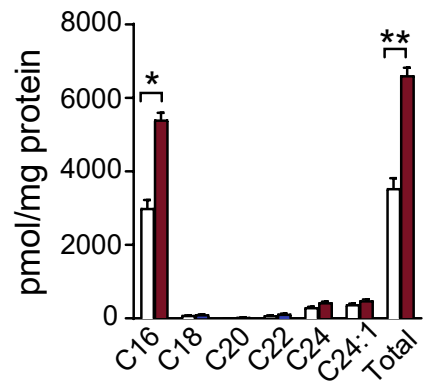

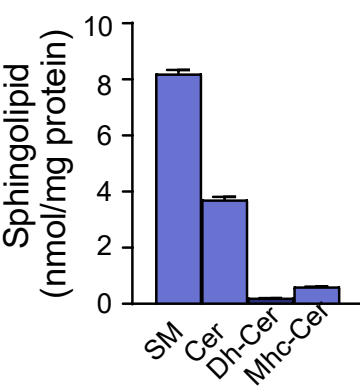
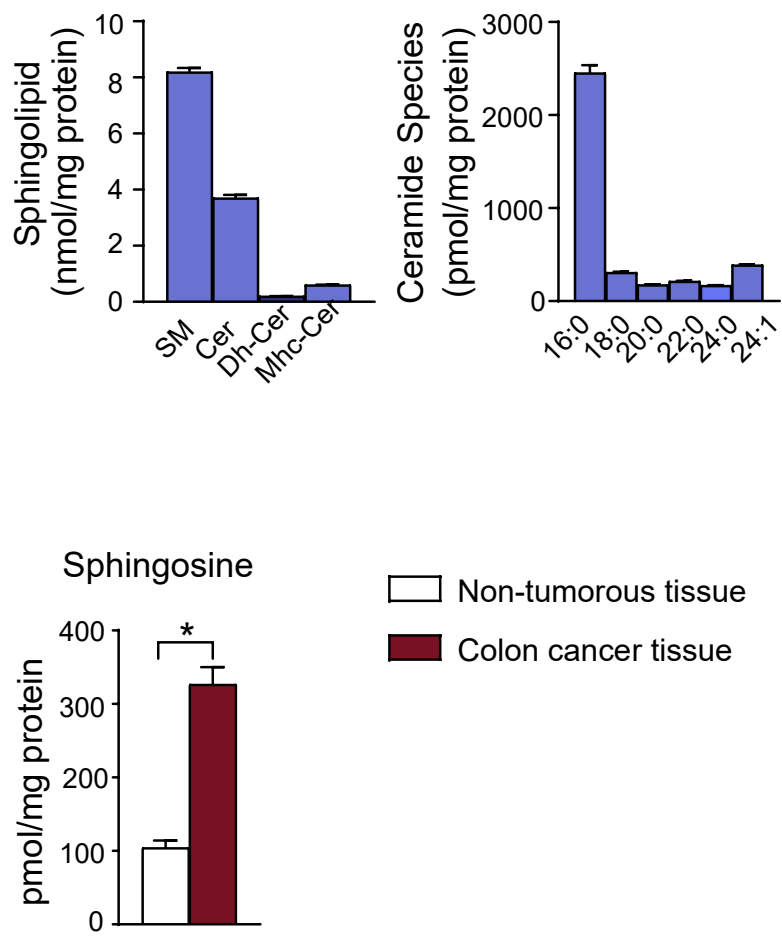

E

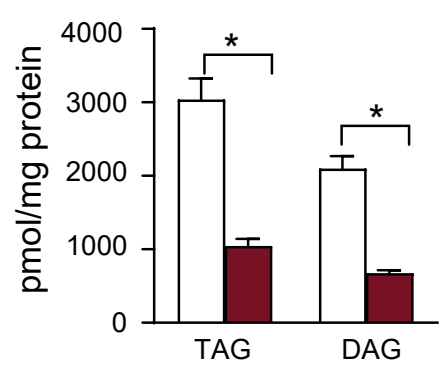


Figure 2

A

B

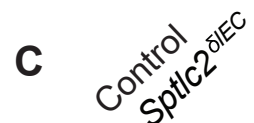

- Control

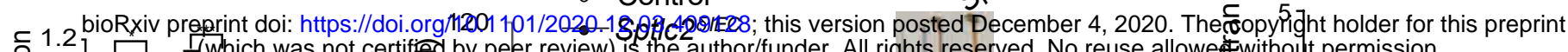
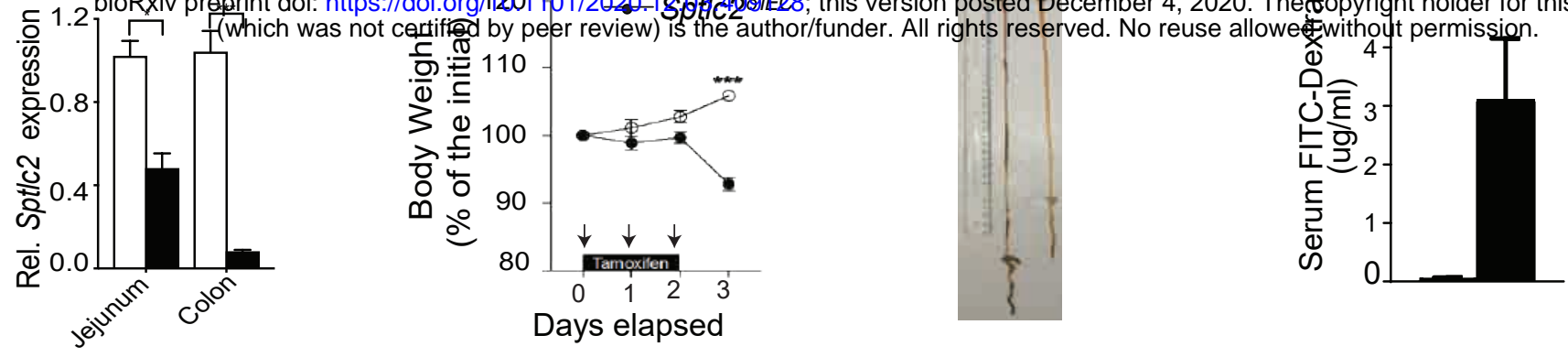

E

Control

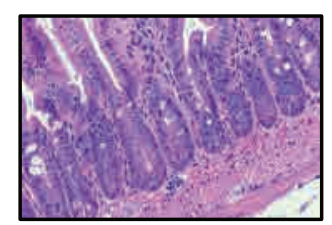

Spt/c2 ${ }^{\delta I E C}$

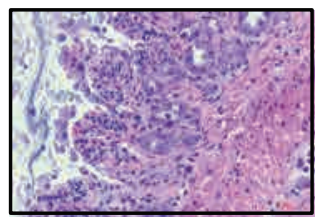

G

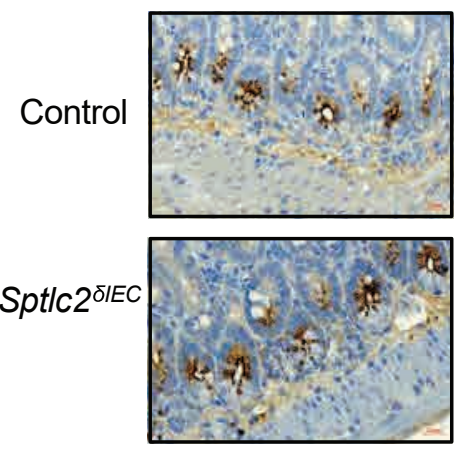

F
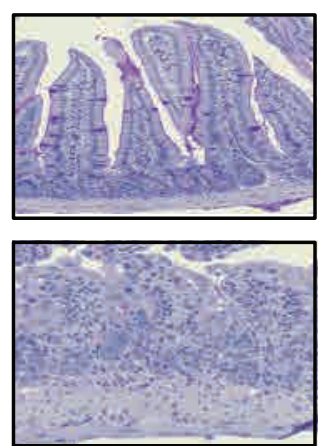

Day1
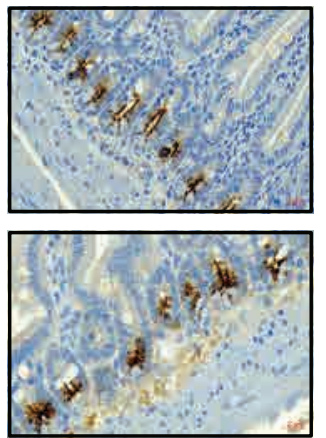

LYZ
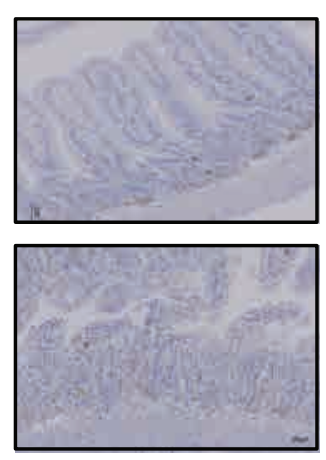

Day2
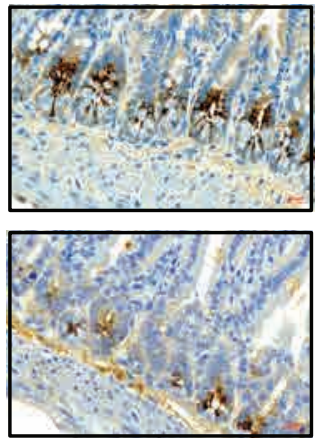

Day3
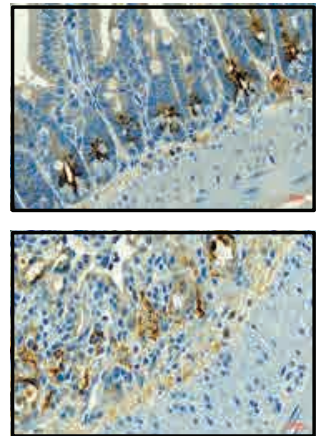

OLFM4
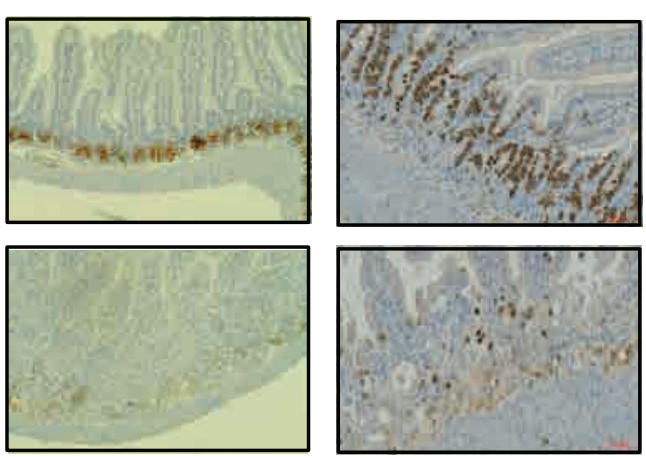

H

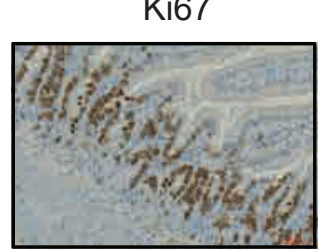

I
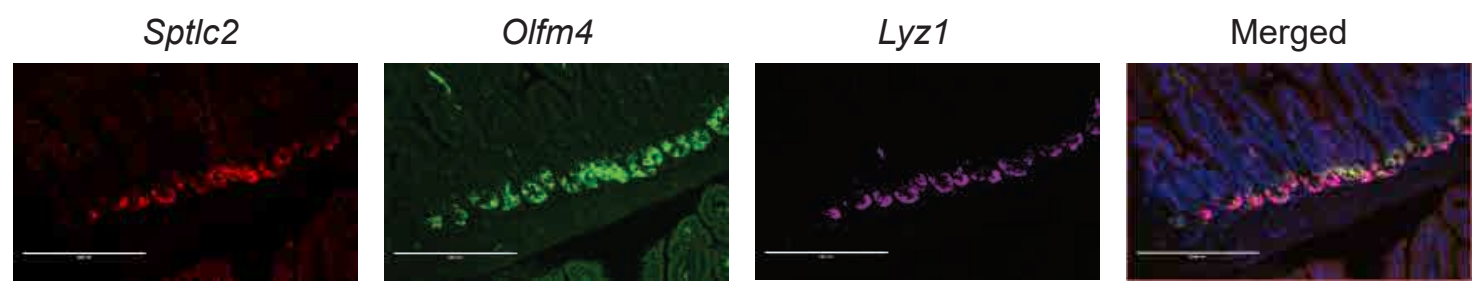

Spt/c2 $2^{\delta / S C}$
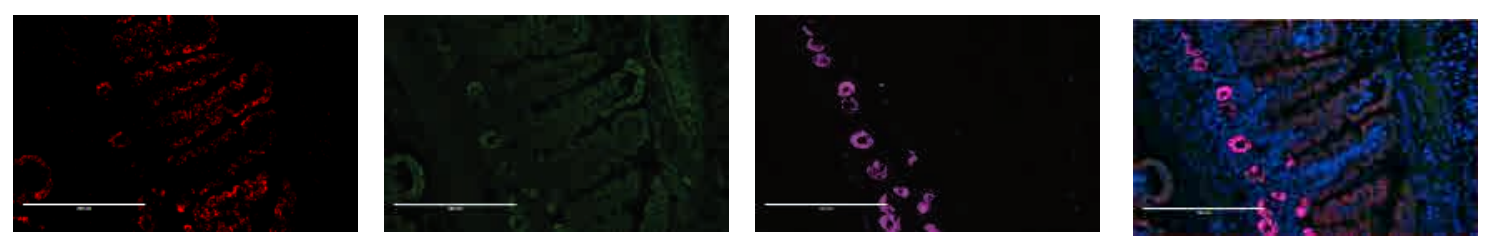

$\mathbf{J}$

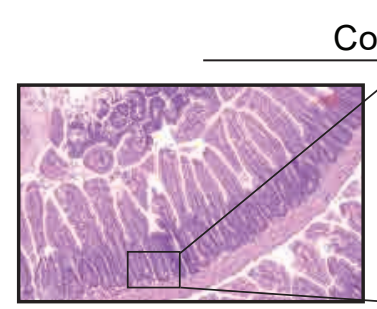

20X
Control

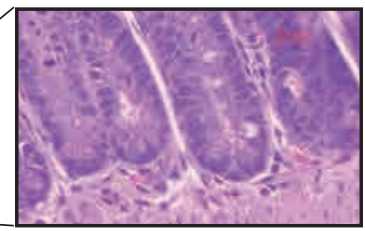

$60 x$

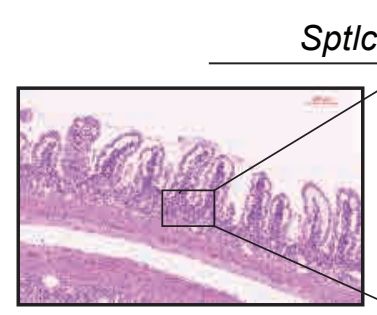

$20 X$

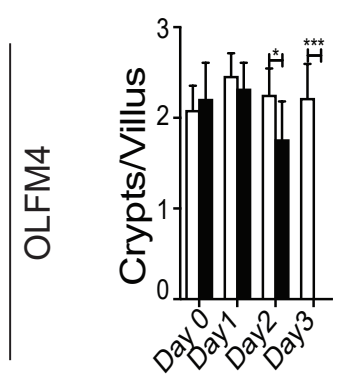

Spt/c $2^{\delta / S C}$

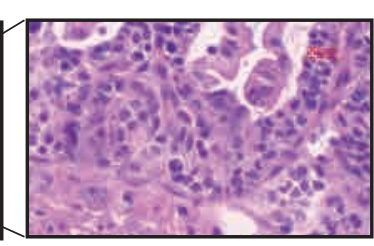

$60 X$ 
Figure 3

A

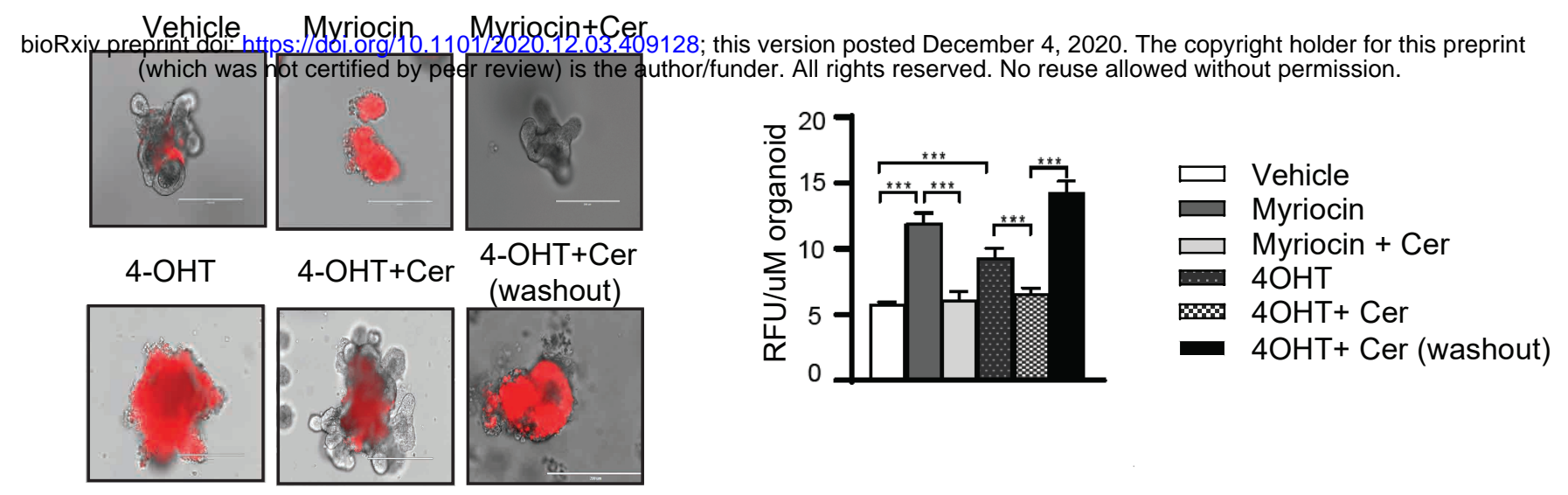

B
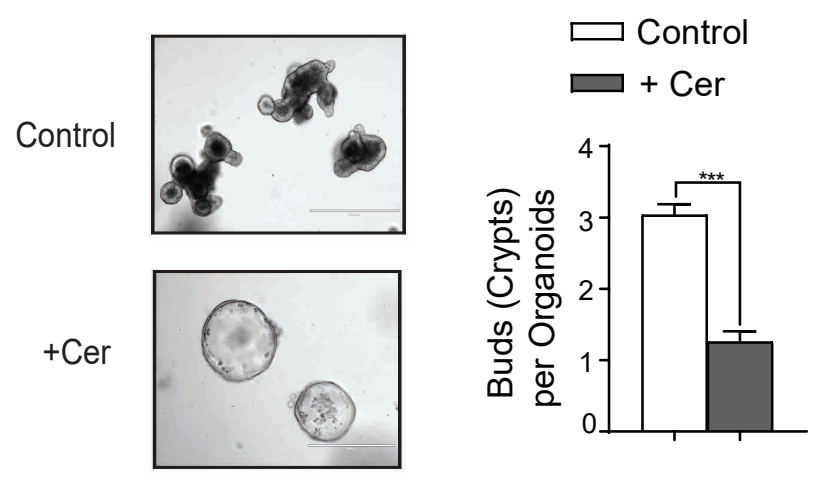

C
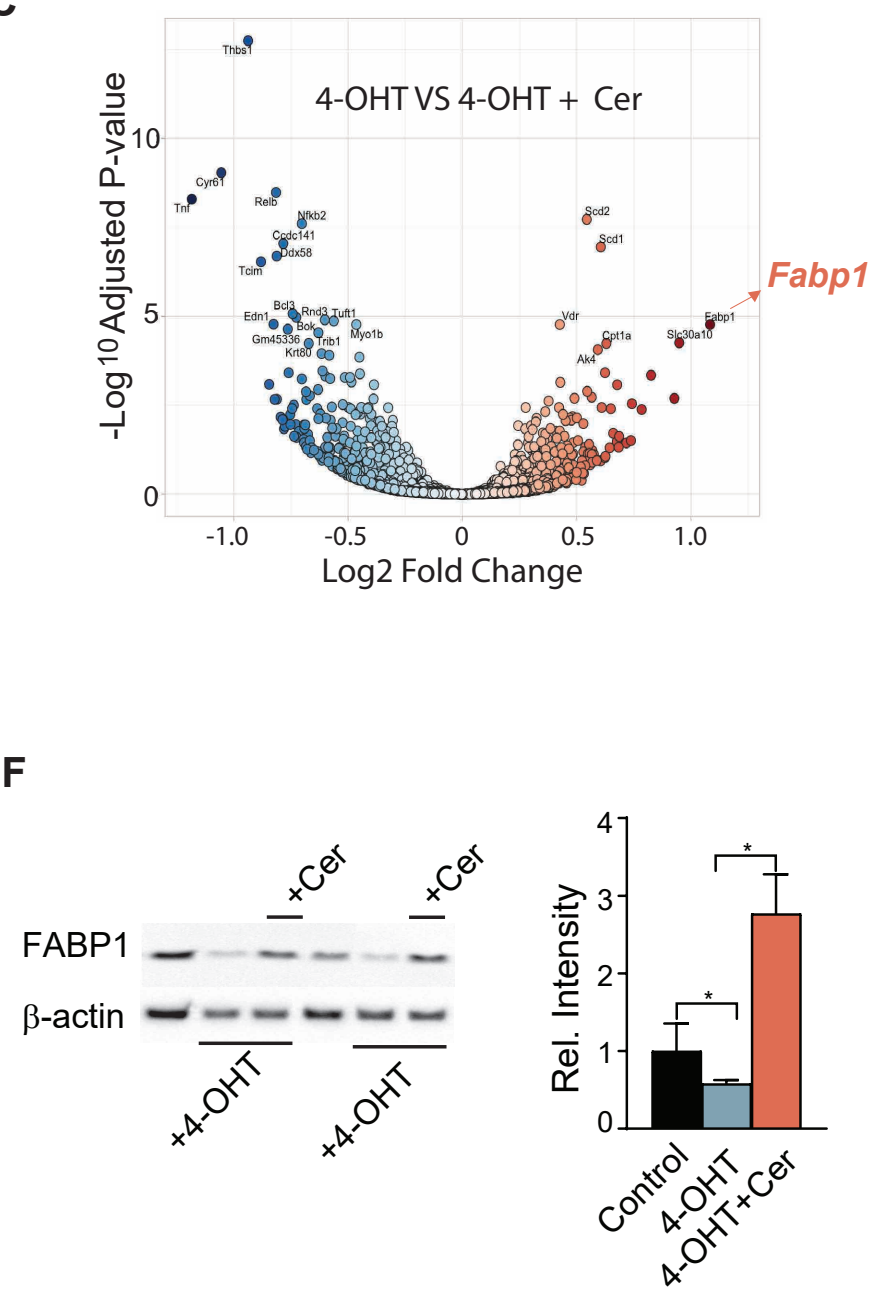
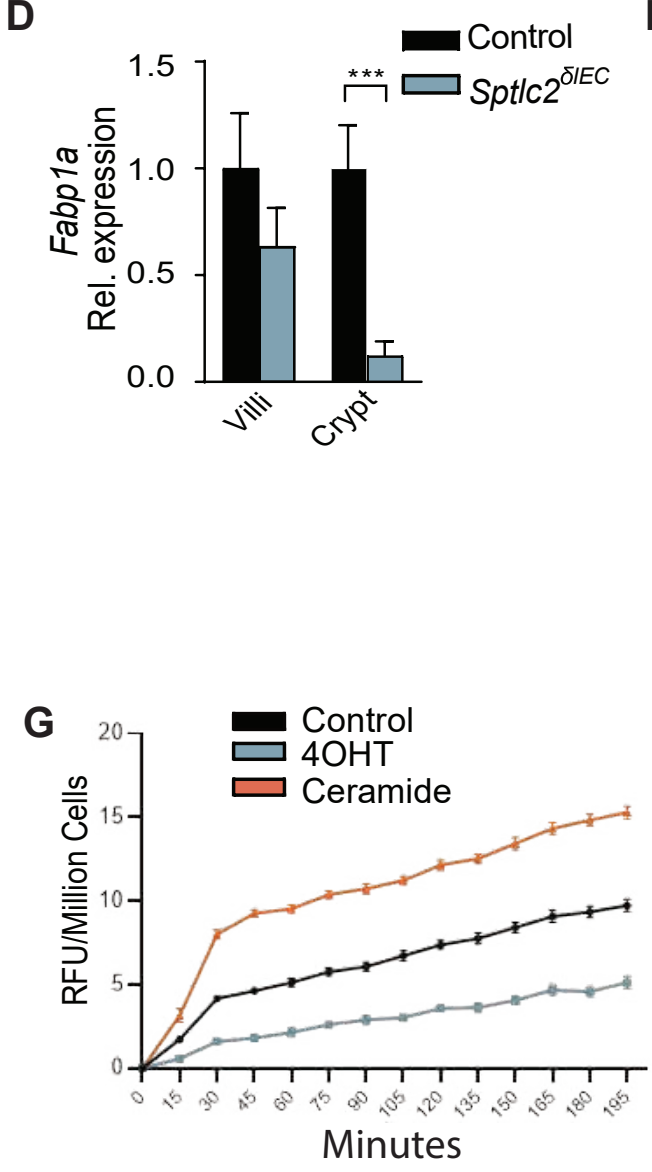

F
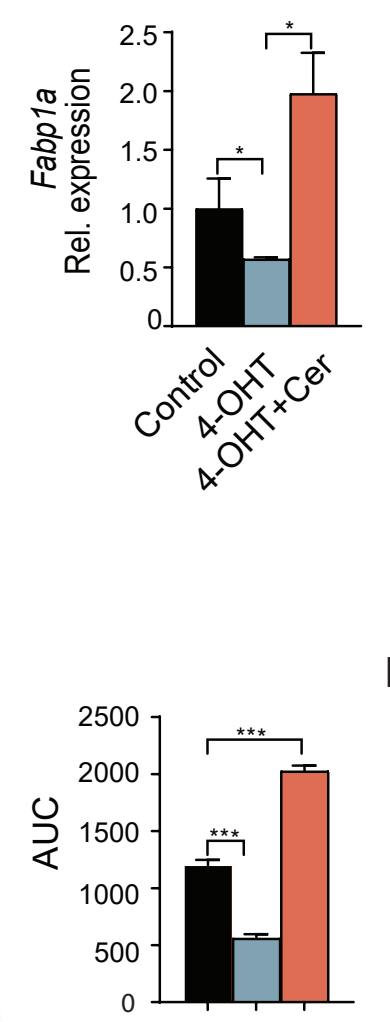

E

H

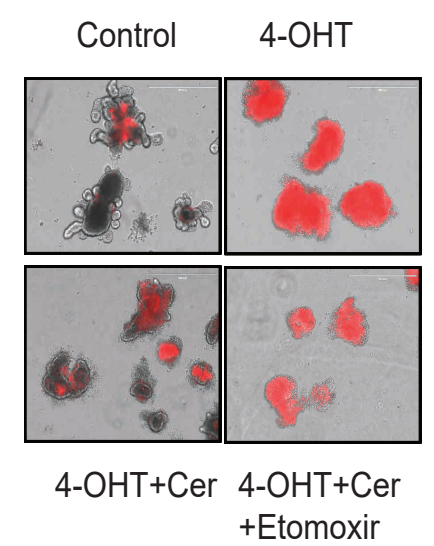

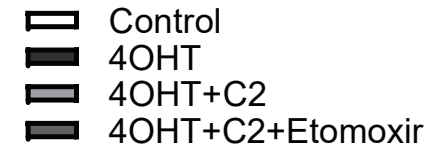

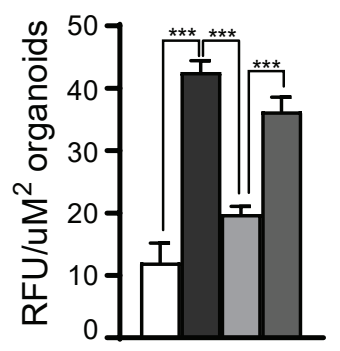


Figure 4

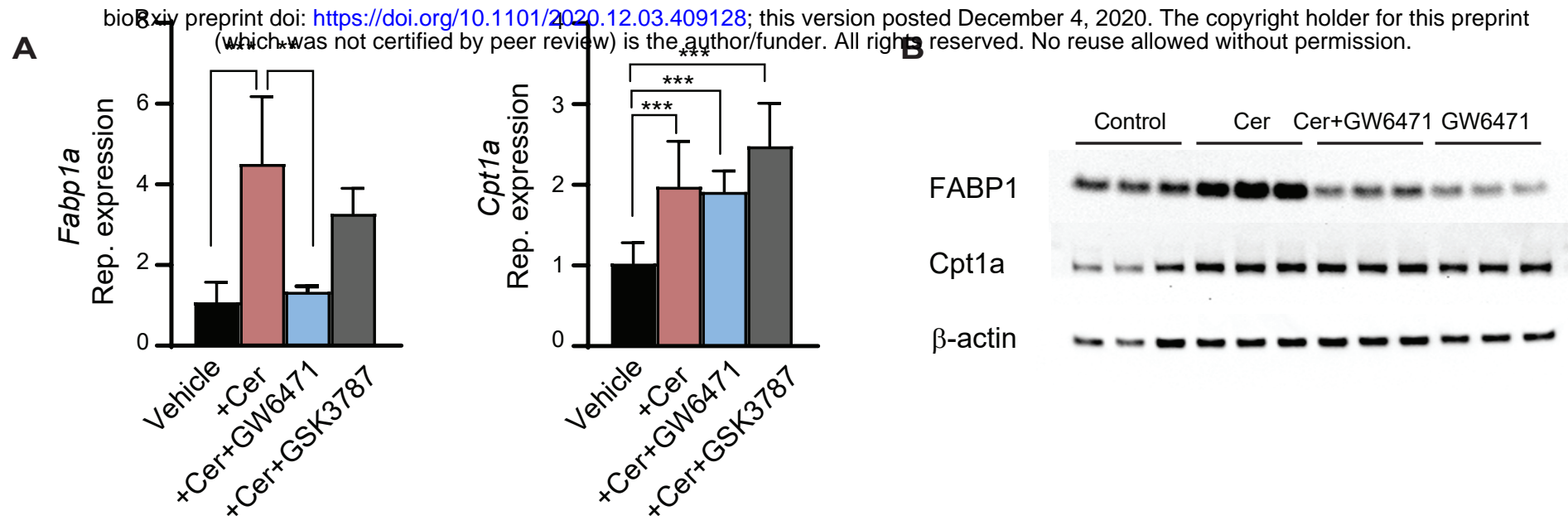

C
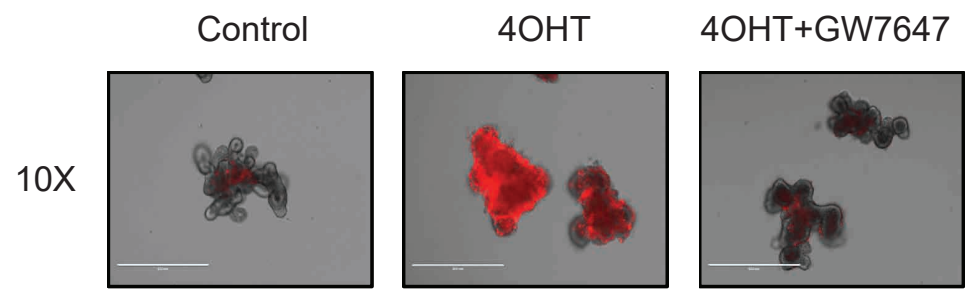

$40 x$
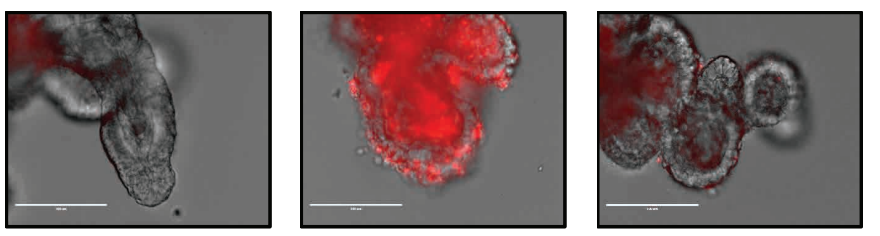

E

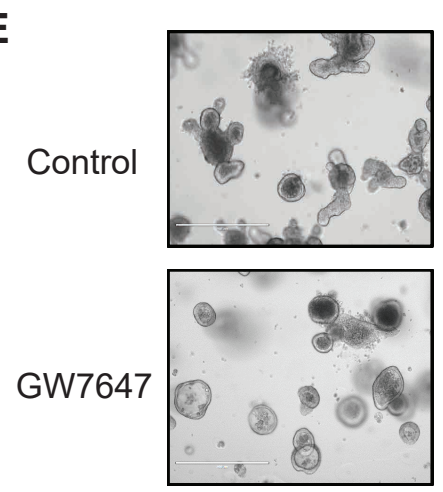

F

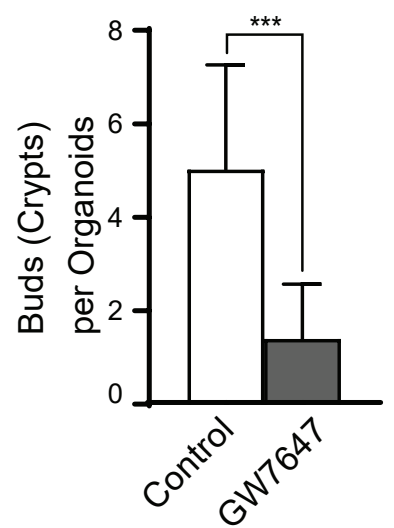

D

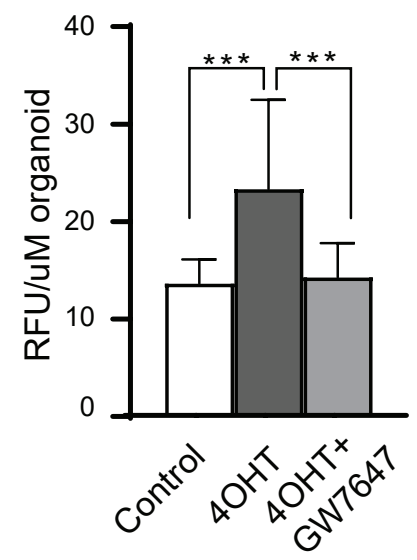

G

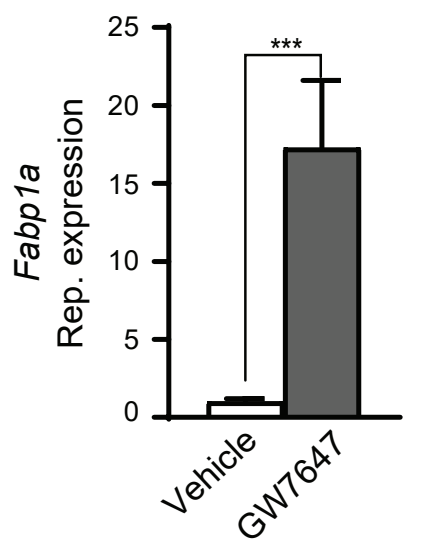

H

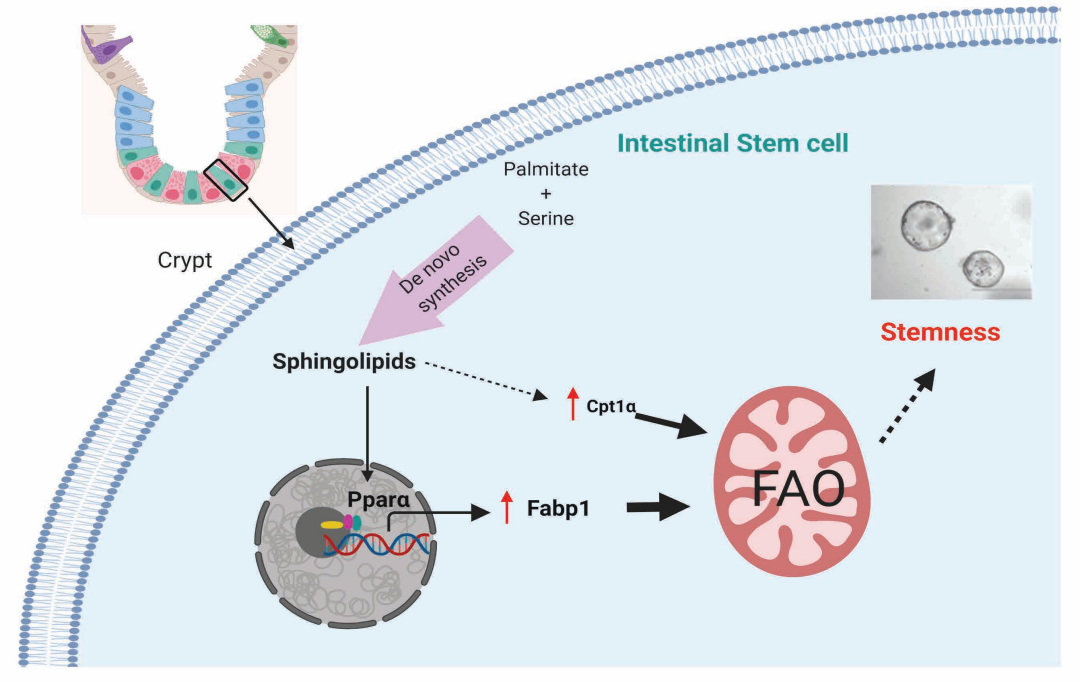


Figure S1. Related to Figure 1.

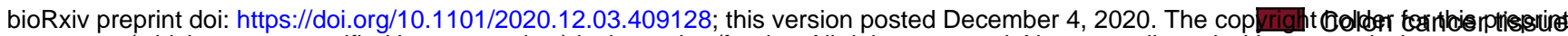
(which was not certified by peer review) is the author/funder. All rights reserved. No reuse allowed without permission.

A

Sphingomyelins

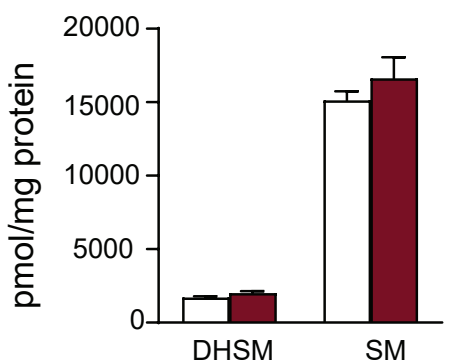

Sphinganine

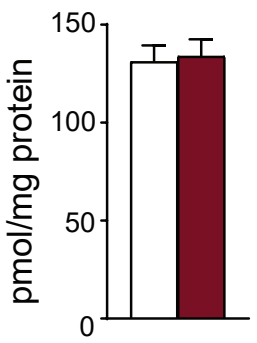

Phytoceramides

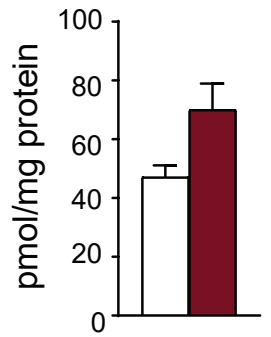

Glucosylceramides

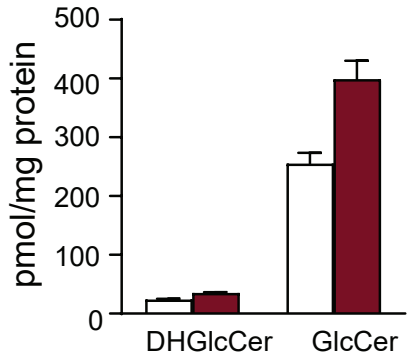

B

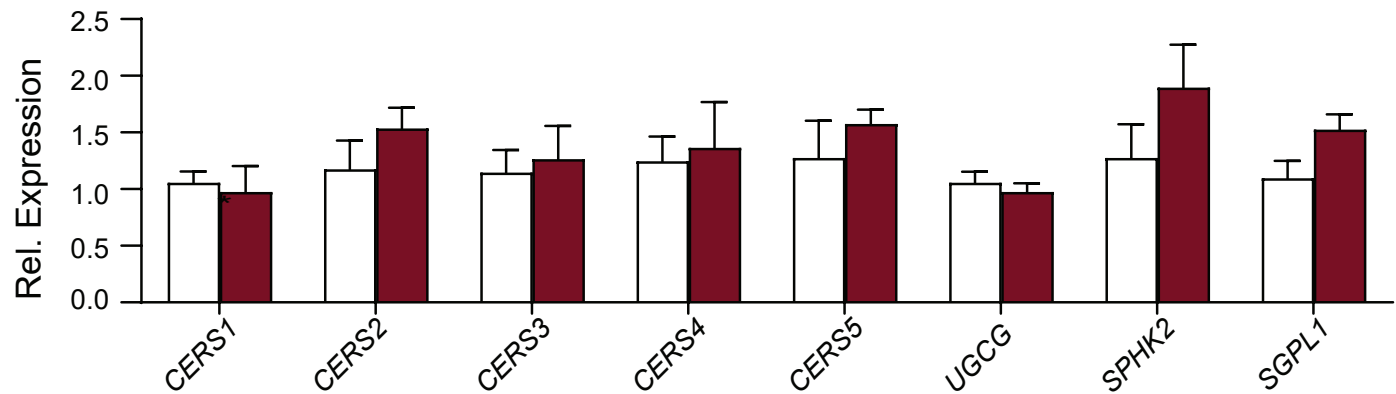

C

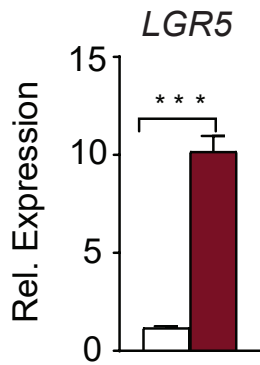


Figure. S1 (related to Figure 1). Quantification of Sphingolipids in Colorectal Cancer. Quantification of (A) sphingolipids, (B) transcripts encoding genes involved in sphingolipid metabolism (using qPCR), and (C) transcripts encoding LGR5 in colon cancer tissue and adjacent non-tumorous tissue. $(\mathrm{n}=9)(* \mathrm{p}<0.05, * * \mathrm{p}<0.01, * * * \mathrm{p}<0.001, \mathrm{n}=9)$. Abbreviations: DHSM, dihydrosphingomyelin; SM, sphingomyelin; DHGlcCer, dihydroglucosylceramide; GlcCer, glucosylceramide; CERS1, ceramide synthase-1; CERS2, ceramide synthase-2; CERS3, ceramide synthase-3; CERS4, ceramide synthase-4; CERS5, ceramide synthase-5; UGCG, Ceramide glucosyltransferase; SPHK2, sphingosine kinase 2; SGPL1, sphingosine phosphate lyase 1; LGR 5, Leucine-rich repeat-containing G-protein coupled receptor 5.Type or paste caption here. Create a page break and paste in the Figure above the caption. 
Figure S2. Related to Figure 2.

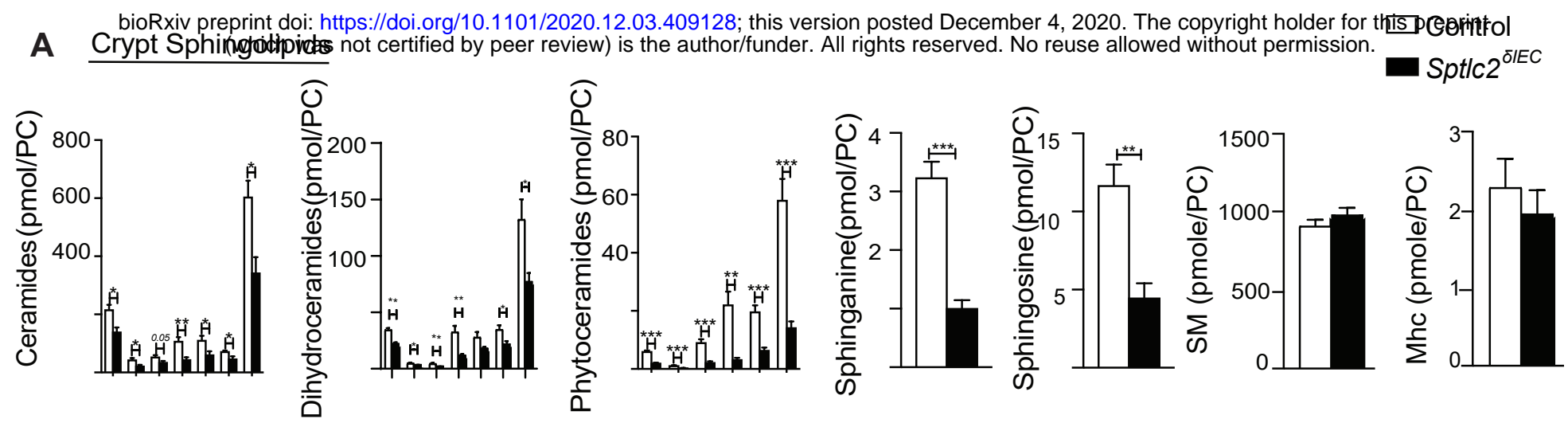

B Villus Sphingolipids

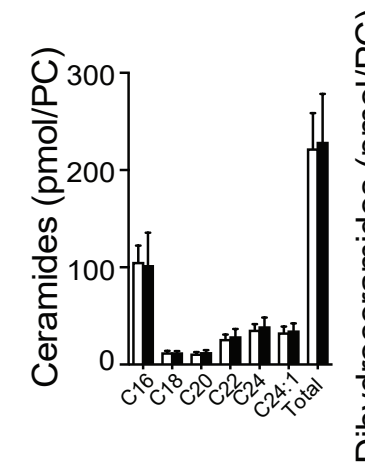

C

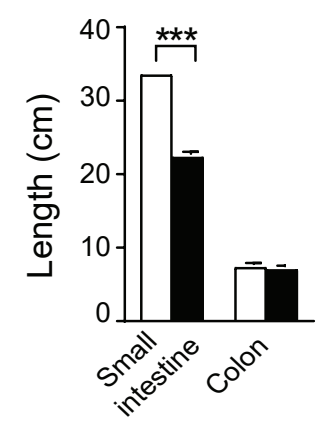

Control Spttc2 $2^{\delta I E C}$

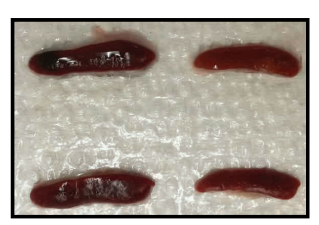

Day0
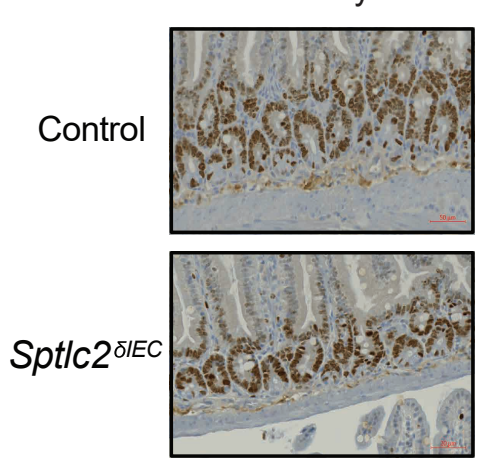

G

Control
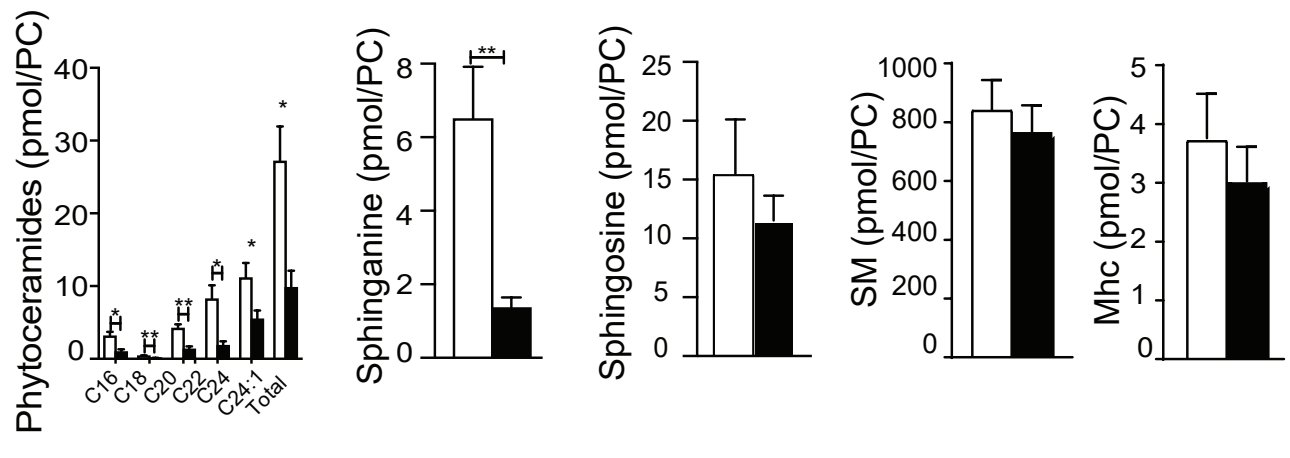

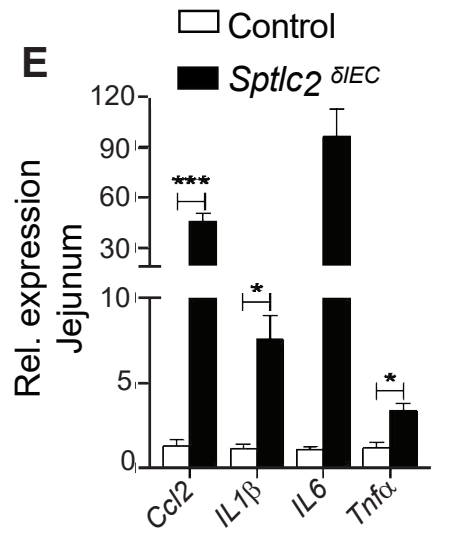

Day2
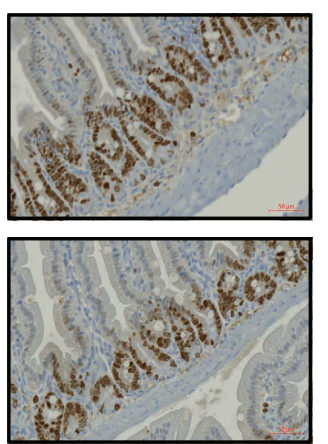
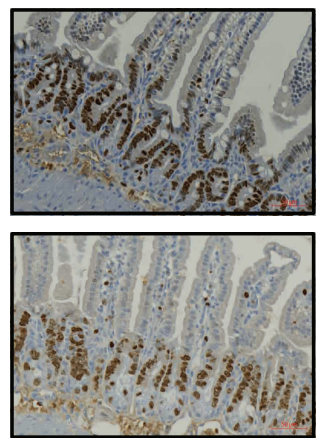

F

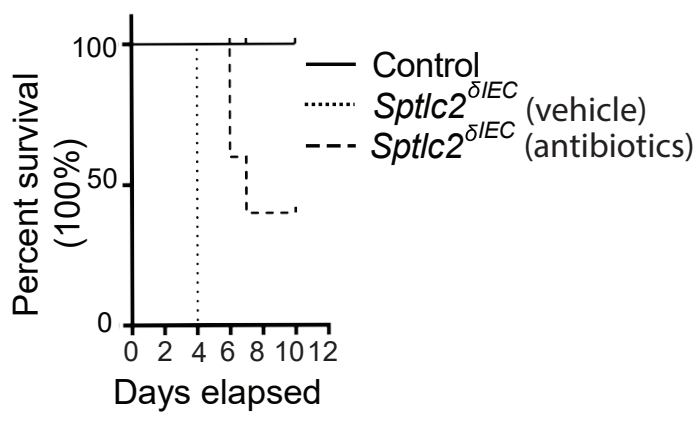

Day3

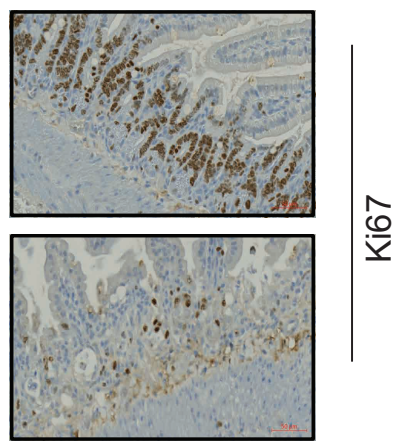

H

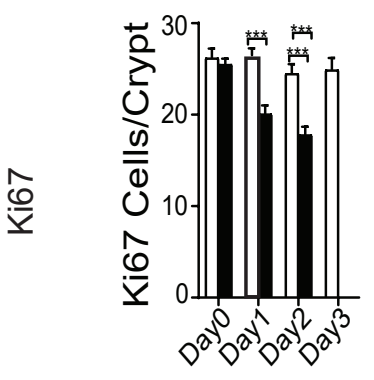


Figure. S2 (related to Figure 2). Deletion of Sptlc2 from intestines disrupts the epithelium and depletes intestinal stem cells. (A) Quantification of sphingolipids in the crypt from the jejunum of Sptlc2 $2^{\delta I E C}$ and $S p t l c^{f l f l}$ littermates 2-days after mice received the first tamoxifen dose. (B) Quantification of sphingolipids in villi isolated from the jejuna of Sptlc2 $\delta$ IEC and Sptlcfl/fl littermates two days after tamoxifen treatment. (C) Quantification of small intestine length in Sptlc $\delta I E C$ and Sptlc2fl/fl littermates four days after tamoxifen treatment $(n=6)$. (D) Image of spleens harvested four days after tamoxifen treatment. (E) qPCR analysis of inflammatory markers four days after tamoxifen injection. (F) Survival curve of Sptlc2 ${ }^{\delta I E C}$ and $S p t l c^{f l / f l}$ mice treated with or without antibiotics. (G) Time course study showing Ki67 positive cells in the small intestine from Sptlc2 $2^{\delta I E C}$ and Sptlc ${ }^{f l f l}$ mice. (H) Quantification of Ki67 positive cells in each crypt. Abbreviations: PC, phosphatidylcholine; IEC, intestinal epithelia cell; SM, sphingomyelin; Mhc, monohexosylceramide; Ccl2, chemokine ligand 2; IL1, interleukin 1-beta; IL6, interleukin 6; Tnf, tumor necrosis factor alpha; H\&E, Hematoxylin and Eosin staining. 
Figure S3. Related to Figure 2.

A

bioRxiv preprint doi: https://doi.org/10.1101/2020.12.03.409128; this version posted December 4, 2020. The copyright holder for this preprint (which was not certified by peer review) is the author/funder. All rights reserved. No reuse allowed without permission.

B

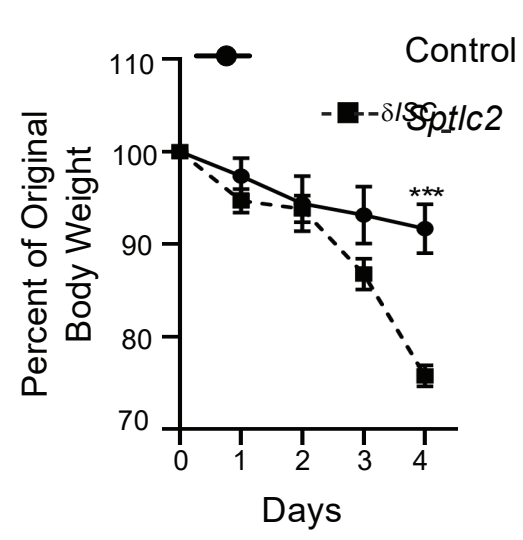

$E$

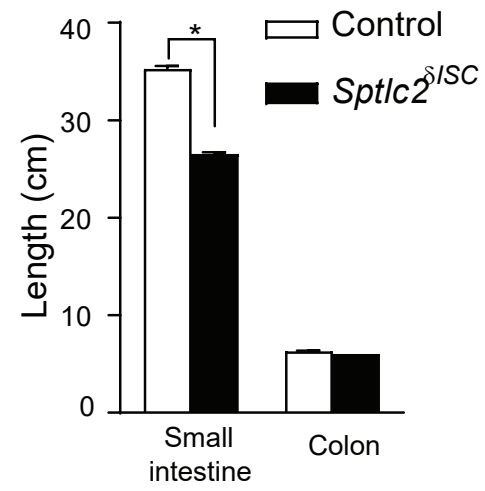
intestine
C

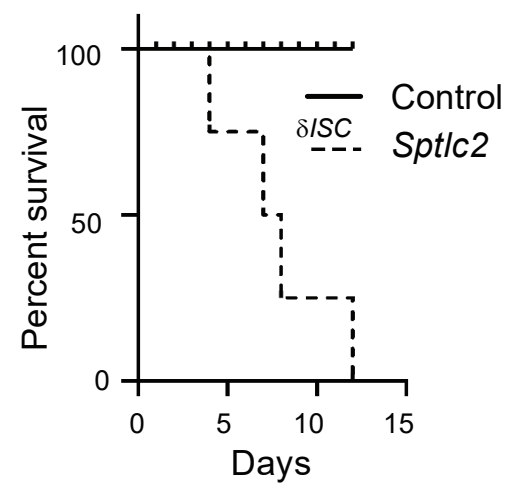

D

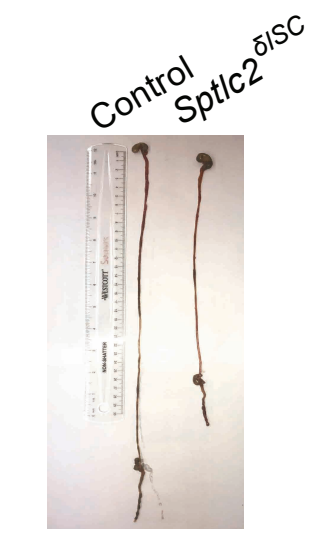

$\mathbf{F}$

Control Spt/c2 $2^{\delta / S C}$
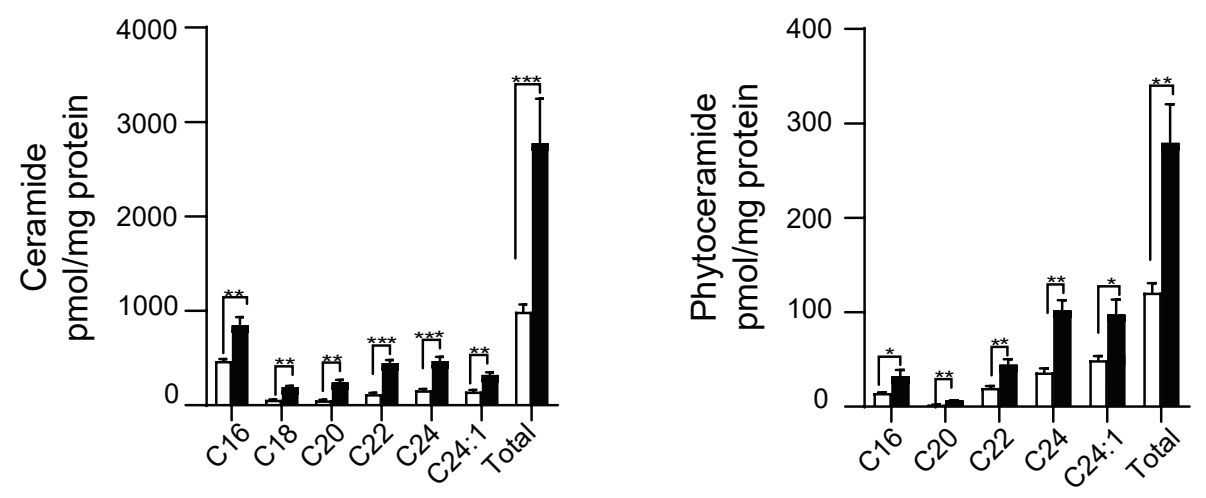

$\square$ Villi

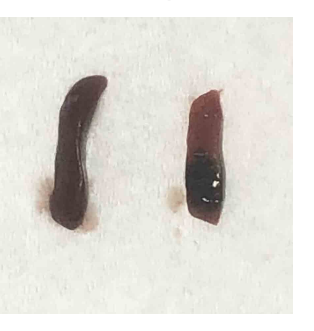


Figure. S3 (related to Figure 2). Deletion of Sptlc2 from intestinal stem cells (Sptlc $\delta$ ISC) recapitulates the pathologic phenotype of the Sptlc $\delta$ IEC mice. (A) Quantification of sphingolipids in the villi or crypt of small intestine from a wide type C57BL/6 mouse. (B) Body weight of mice after Sptlc2 gene deletion. (C) Survival curve of Sptlc2 $2^{\delta I S C}$ and Sptlc ${ }^{\text {flff }}$ littermates. (D) Image of small intestine after Sptlc2 deletion. (E) Quantification of the length of the intestines. (F) Image of spleens harvested four days after tamoxifen treatment. Abbreviations. ISC, intestinal stem cell; H\&E, Hematoxylin and Eosin staining. 
Figure S4. Related to Figure 4

A bioRxiv preprint doi: https://doi.org/10.1101/2020.12.03.409128; this version posted December 4, 2020. The copyright hold Control

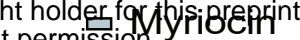

Ceramides

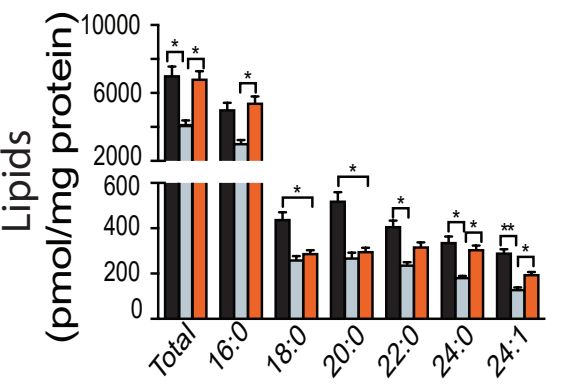

B

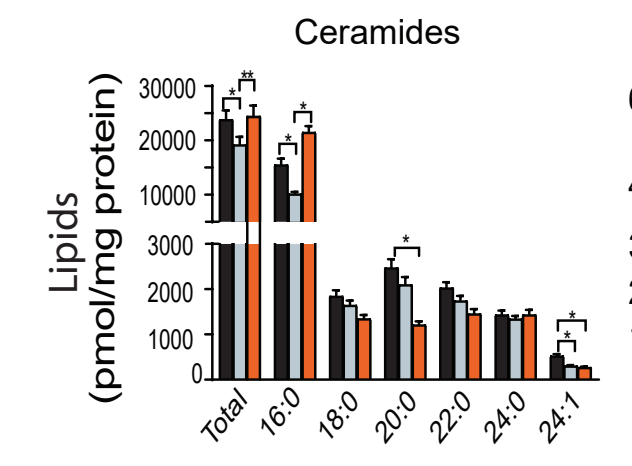

$\mathbf{B}$
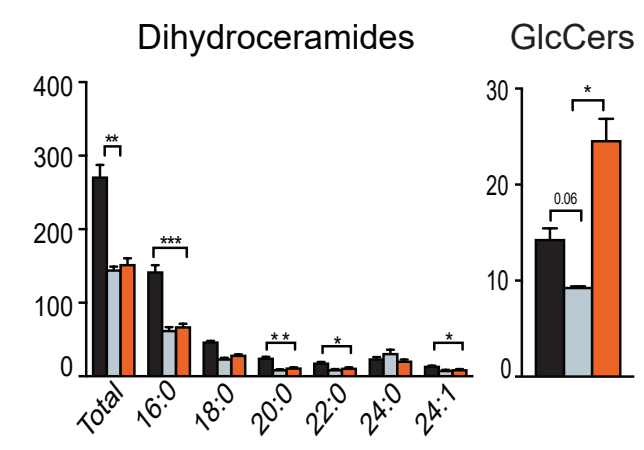

Myriocin + Cer

Dihydroceramides

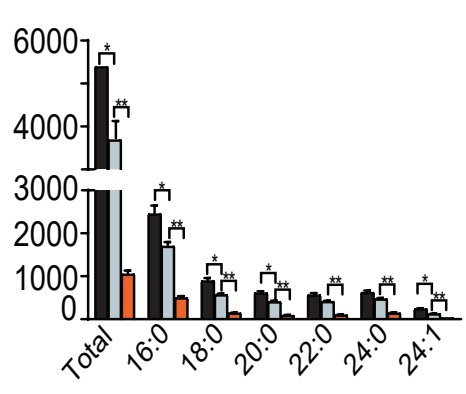

GlcCers

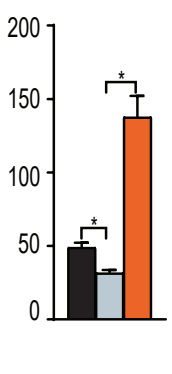

SMs
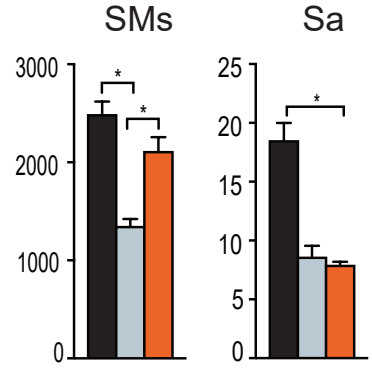

Sa

So

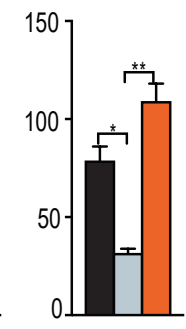

- Control

$\square$ 4-OHT

- 4-OHT + Cer
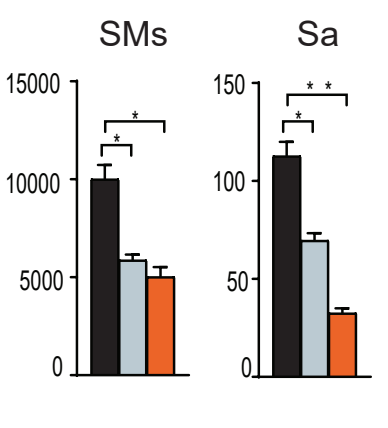
So

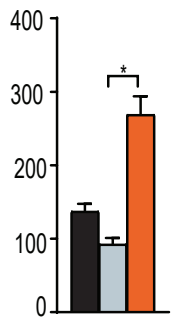

C
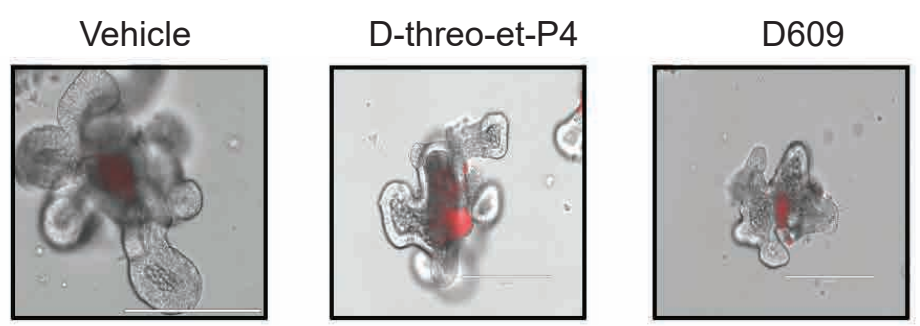
Figure. S4 (related to Figure 3 and 4). Quantification of Sphingolipids in Organoids. (A) Quantification of sphingolipids in organoids treated with vehicle, myriocin $(10 \mu \mathrm{M})$, or myriocin plus C2-ceramide $(25 \mu \mathrm{M})$ for 24 hours. (B) Quantification of sphingolipids in organoids treated with vehicle, $4 \mathrm{OHT}(200 \mathrm{ng} / \mathrm{ml})$ or $4 \mathrm{OHT}$ plus C2 ceramide $\left(\mathrm{n}=6,{ }^{*} \mathrm{p}<0.05,{ }^{*} \mathrm{p}<0.01\right.$, $* * * p<0.001)$. (C) PI staining of organoids treated with vehicle, De-threo-et-P4 (100nM, GCS inhibitor) or D609 (100nM, SMS inhibitor) for 72 hours. Abbreviations: GlcCers, glucosylceramides; SMs, sphingomyelins; Sa, sphinganine; So, sphingosine; Cer, Ceramide (d18:1/2:0), N-acetyl-D-erythro-sphingosine; 4OHT, 4-hydroxytamoxifen. 